\title{
Poles of Igusa's local zeta function and monodromy
}

Bulletin de la S. M. F., tome 121, no 4 (1993), p. 545-598

<http://www.numdam.org/item?id=BSMF_1993_121_4_545_0>

(C) Bulletin de la S. M. F., 1993, tous droits réservés.

L'accès aux archives de la revue «Bulletin de la S. M. F. » (http: //smf.emath.fr/Publications/Bulletin/Presentation.html) implique l'accord avec les conditions générales d'utilisation (http://www.numdam.org/ conditions). Toute utilisation commerciale ou impression systématique est constitutive d'une infraction pénale. Toute copie ou impression de ce fichier doit contenir la présente mention de copyright.

\section{Numdam}


Bull. Soc. math. France, 121, 1993, p. 545-598.

POLES OF IGUSA'S LOCAL ZETA FUNCTION AND MONODROMY

BY

\author{
WILLEM VEYS
}

RÉSUMÉ. - Soit $K$ une extension finie de $\mathbb{Q}_{p}$ et $R$ son anneau de valuation. On associe à chaque $f \in K[x]$, avec $x=\left(x_{1}, \ldots, x_{n}\right)$, la fonction zêta locale d'Igusa

$$
Z(s)=\int_{R^{n}}|f(x)|^{s}|\mathrm{~d} x|,
$$

qui est méromorphe sur $\mathbb{C}$. La conjecture de monodromie associe des valeurs propres de la monodromie (complexe) de l'hypersurface $f=0$ aux pôles de $Z(s)$. On peut exprimer une liste de candidats-pôles de $Z(s)$ ainsi que les valeurs propres de la monodromie à l'aide de données numériques de variétés exceptionelles, associées à une résolution plongée de $f=0$. En utilisant des relations entre ces données numériques on montre que certains candidats-pôles ne contribuent pas aux vrais pôles, ce qui entraîne une forte évidence concernant la conjecture.

Abstract. - Let $K$ be a finite extension of $\mathbb{Q}_{p}$ and $R$ its valuation ring. To any $f \in K[x]$, with $x=\left(x_{1}, \ldots, x_{n}\right)$, is associated Igusa's local zeta function

$$
Z(s)=\int_{R^{n}}|f(x)|^{s}|\mathrm{~d} x|,
$$

which is known to be meromorphic on $\mathbb{C}$. The monodromy conjecture relates poles of $Z(s)$ to eigenvalues of the (complex) monodromy of the hypersurface $f=0$. Now we can express both a list of candidate-poles for $Z(s)$ and the monodromy-eigenvalues in terms of certain numerical data of exceptional varieties, associated to an embedded resolution of $f=0$. Using relations between those numerical data we study the vanishing of bad candidate-poles for $Z(s)$ to obtain a lot of evidence for the conjecture.

$\left({ }^{*}\right)$ Texte reçu le 23 juillet 1992.

W. VEYs, K.U. Leuven, Departement Wiskunde, Celestijnenlaan 200B, B-3001 Leuven, Belgium.

The author wants to thank the Department of Mathematics of the École Polytechnique at Palaiseau for its support during the preparation of this manuscript.

AMS classification : 11 S 40, 14G10.

BULLETIN DE LA SOCIÉTÉ MATHÉMATIQUE DE FRANCE 0037-9484/1993/545/\$5.00

(C) Société mathématique de France 


\section{Introduction}

Let $K$ be a number field and $R$ its ring of algebraic integers. For any maximal ideal $\mathfrak{p}$ of $R$, let $R_{\mathfrak{p}}$ and $K_{\mathfrak{p}}$ denote the completion of respectively $R$ and $K$ with respect to the $\mathfrak{p}$-adic absolute value. Let $|x|$ denote this absolute value for $x \in K_{\mathfrak{p}}$, and let $q$ be the cardinality of the residue field $\bar{K}=R_{\mathfrak{p}} / \mathfrak{p} R_{\mathfrak{p}}$. (For example if $K=\mathbb{Q}$ we have that $\mathfrak{p}$ is determined by a prime number $p$, then $K_{\mathfrak{p}}$ is the field of $p$-adic numbers $\mathbb{Q}_{p}$ and $\bar{K}$ is the finite field with $p$ elements.)

Let $f(x) \in K[x]$, with $x=\left(x_{1}, \ldots, x_{n+1}\right)$. Then Igusa's local zeta function of $f$ is defined by

$$
Z(s)=Z_{\mathfrak{p}}(s)=\int_{R_{\mathfrak{p}}^{n+1}}|f(x)|^{s}|\mathrm{~d} x|,
$$

where $|\mathrm{d} x|$ denotes the Haar measure normalized such that $R_{\mathfrak{p}}^{n+1}$ has measure one. It describes the Poincaré series

$$
P(T)=\sum_{i=0}^{\infty} N_{i}\left(q^{-(n+1)} T\right)^{i}
$$

where $N_{i}$, with $i \in \mathbb{N}$, is the number of solutions of $f=0$ in the ring $R_{\mathfrak{p}} / \mathfrak{p}^{i} R_{\mathfrak{p}}$, through the relation

$$
Z(s)=\left(1-q^{s}\right) P\left(q^{-s}\right)+q^{s} .
$$

IgusA $[\operatorname{Ig} 1]$ proved that $Z(s)$, and therefore also $P(T)$, is a rational function of $q^{-s}=T$.

One can compute $Z(s)$ using an embedded resolution with normal crossings for $f=0$ in $\mathbb{A}^{n+1}\left(\mathbb{Q}^{a}\right)$, where $\mathbb{Q}^{a}$ is the algebraic closure of $\mathbb{Q}$. (An explicit formula of Denef [D1] is stated in Theorem 1.2.) Let $(X, h)$ be such a resolution, obtained by Hironaka's main theorem [Hi], and denote by $E_{i}$, with $i \in S$, the (reduced) irreducible components of $h^{-1}\left(f^{-1}\{0\}\right)$. We associate to each $E_{i}, i \in S$, a pair of numerical data $\left(N_{i}, \nu_{i}\right)$ where $N_{i}$ and $\left(\nu_{i}-1\right)$ are the multiplicities of $E_{i}$ in the divisor of respectively $f \circ h$ and $h^{*}\left(\mathrm{~d} x_{1} \wedge \cdots \wedge \mathrm{d} x_{n+1}\right)$ on $X$.

In particular all real poles of $Z(s)$ are part of the set $\left\{-\nu_{i} / N_{i} \mid i \in S\right\}$. So determining the real poles consists in throwing away the bad candidates. Now it is striking that «most» candidate-poles are actually bad. This fact would be elucidated if the following monodromy conjecture is true. 
Conjecture. - For all except a finite number of $\mathfrak{p}$ we have that, if $s$ is a pole of $Z_{\mathfrak{p}}(s)$, then $\mathrm{e}^{2 \pi i \operatorname{Re}(s)}$ is an eigenvalue of the monodromy acting on the cohomology (in some dimension) of the Milnor fiber of $f$ associated to some point of the hypersurface $f=0$.

We explain this more in detail. (For the concept of monodromy we refer to Milnor [Mi]). Fix an exceptional variety $E_{j}$ and set $\stackrel{\circ}{E}_{j}=$ $E_{j} \backslash\left(\bigcup_{i \neq j} E_{i}.\right)$ For any scheme $V$ of finite type over $K$ let $\chi(V)$ denote the Euler-Poincaré characteristic of $V(\mathbb{C})$. Suppose that $\nu_{j}$ and $N_{j}$ are coprime and that there is no $E_{i}$, with $i \in S \backslash\{j\}$, with $N_{j} \mid N_{i}$. The monodromy conjecture implies, for all except a finite number of prime ideals $\mathfrak{p}$, that $s=-\nu_{j} / N_{j}$ is no pole of $Z(s)$ if $\chi\left(\stackrel{\circ}{E}_{j}\right)=0$. (We illustrate this in paragraph 2.) Now in any concrete example we have that $\chi\left(\stackrel{\circ}{E}_{j}\right)=0$ for «most» exceptional varieties $E_{j}$.

IGUSA [Ig5] tested the monodromy conjecture for relative invariants of certain reductive groups. LOESER verified it for arbitrary polynomials in two variables [L1], and for polynomials which are non-degenerate with respect to their Newton polyhedron, assuming certain additional conditions [L3]. We should also mention that the archimedean analogon of the conjecture has been proved by MaLGRANGE [Ma1], [Ma2].

In this paper we are interested in the vanishing of bad candidate-poles for $Z(s)$ to obtain more evidence for the monodromy conjecture, using relations between the numerical data of the resolution $(X, h)$ for $f=0$. Considering the formula for $Z(s)$ of THEOREM 1.2, it is clear that relations between the numerical data of $E_{j}$ and of the $E_{i}, i \in S \backslash\{j\}$, that intersect $E_{j}$ are very useful to make conclusions about the residue of $-\nu_{j} / N_{j}$.

Relations. - In [V2] we proved for arbitrary polynomials $f$ relations between numerical data, which we state briefly in paragraph 0 . We now explain the essential aspects of those relations.

Fix one exceptional variety $E$ with numerical data $(N, \nu)$. The variety $E$ in the final resolution $X$ is in fact obtained by a finite succession of blowing-ups

$$
E^{0} \stackrel{\pi_{0}}{\longleftarrow} E^{1} \stackrel{\pi_{1}}{\longleftarrow} \cdots E^{i} \stackrel{\pi_{i}}{\longleftarrow} E^{i+1} \ldots \stackrel{\pi_{m-2}}{\longleftarrow} E^{m-1} \stackrel{\pi_{m-1}}{\longleftarrow} E^{m}=E
$$

with irreducible nonsingular center in $E^{i}$ and exceptional variety $C_{i+1} \subset$ $E^{i+1}$ for $i=0, \ldots, m-1$. The variety $E^{0}$ is created at some stage of the global resolution process as the exceptional variety of a blowing-up with center $D$ and is isomorphic to a projective space bundle $\Pi: E^{0} \rightarrow D$ over $D$. 
There are two kinds of intersections of $E$ with other components of $h^{-1}\left(f^{-1}\{0\}\right)$. We have the repeated strict transforms $C_{1}^{(m)}, \ldots, C_{m}^{(m)}$ in $E$ of the exceptional varieties $C_{1}, \ldots, C_{m}$; and furthermore we have the repeated strict transforms $C_{i}^{(m)}$ in $E$ of varieties $C_{i}$, with $i \in T$, (of codimension one) in $E^{0}$.

For each $i \in T \cup\{1, \ldots, m\}$ the strict transform $C_{i}^{(m)}$ of $C_{i}$ in $E$ is (an irreducible component of) the intersection of $E$ with exactly one other component of $h^{-1}\left(f^{-1}\{0\}\right)$. Let this component have numerical data $\left(N_{i}, \nu_{i}\right)$ and set $\alpha_{i}=\nu_{i}-(\nu / N) N_{i}$. (The numbers $\alpha_{i}, i \in T \cup\{1, \ldots, m\}$, occur in the expression for the residue of the candidate-pole $-\nu / N$ for $Z(s)$, see THEOREM 1.2.)

There are basic relations (B1 and B2) between the $\alpha_{i}, i \in T$, and there is for each $i \in\{0, \ldots, m-1\}$ an additional relation (A) expressing $\alpha_{i+1}$ in terms of the $\alpha_{k}$ for $k \in T \cup\{1, \ldots, i\}$.

For the applications on the poles of $Z(s)$, we choose the number field $K$ «large enough», meaning that the resolution $(X, h)$ over $\mathbb{Q}^{a}$ is entirely defined over $K$ itself.

We now suppose that the fixed exceptional variety $E$ satisfies $\chi(\stackrel{\circ}{E})=0$ and that there is no $E_{i}$, with $i \in S \backslash\{j\}$, intersecting $E$ with $\nu_{i} / N_{i}=\nu / N$. Denote by $\mathcal{R}$ the contribution of $E$ to the residue of the candidatepole $-\nu / N$ for $Z(s)$.

Surfaces. - When $n=2$, the surface $E^{0}$ is created by blowing-up a point or a nonsingular curve $D$. In the first case $E^{0} \cong \mathbb{P}^{2}$ and in the latter $E^{0}$ is a ruled surface $\Pi: E^{0} \rightarrow D$ over $D$.

By the formula for $Z(s)$ of ThEOREM 1.2 we can express $\mathcal{R}$ in this case as follows. Set $\stackrel{\circ}{C}_{i}=C_{i}^{(m)} \backslash \bigcup_{\ell \neq i} C_{\ell}^{(m)}$ and $\alpha_{i}=\nu_{i}-(\nu / N) N_{i}$ for $i \in T \cup\{1, \ldots, m\}$. Then

$$
\begin{aligned}
\mathcal{R}=\operatorname{card} \stackrel{\circ}{E} & +(q-1) \sum_{i \in T \cup\{1, \ldots, m\}} \frac{\operatorname{card} \stackrel{\circ}{C}_{i}}{q^{\alpha_{i}}-1} \\
& +(q-1)^{2} \sum_{\substack{\{i, j\} \subset T \cup\{1, \ldots, m\} \\
i \neq j}} \frac{\operatorname{card}\left(C_{i}^{(m)} \cap C_{j}^{(m)}\right)}{\left(q^{\alpha_{i}}-1\right)\left(q^{\alpha_{j}}-1\right)},
\end{aligned}
$$

where card $\stackrel{\circ}{E}$ and card $\stackrel{\circ}{C}_{i}$ are the number of $\bar{K}$-rational points of the reduction of respectively $\stackrel{\circ}{E}$ and $\stackrel{\circ}{C}_{i}$ modulo $\mathfrak{p} R_{\mathfrak{p}}$. 
Using the Relations (A) and (B) we prove for a lot of intersection configurations of the $C_{i}^{(m)}$ on $E$ that $\mathcal{R}=0$ if $\chi(\stackrel{\circ}{E})=0$. We proceed as follows.

By Proposition 2.3 we may suppose that $\bigcup_{i \in T \cup\{1, \ldots, m\}} C_{i}^{(m)}$ in $E$ is the canonical embedded resolution of $\cup_{i \in T} C_{i}$ in $E^{0}$. Therefore the intersection configuration of the $C_{i}^{(m)}, i \in T \cup\{1, \ldots, m\}$, on $E$ is entirely determined by the intersection configuration of the $C_{i}, i \in T$, on $E^{0}$. An important result is then that we can reduce the calculation of $\mathcal{R}$ for a given $C_{i}$-configuration on $E^{0}$ to the calculation of $\mathcal{R}$ for the very easy configuration described in Proposition 2.5. This reduction is possible by a somewhat surprising blowing-down technique (THEOREM 2.4).

Of course the described techniques are only valuable if there exist a lot of (complicated) intersection configurations of the $C_{i}, i \in T$, on $E^{0}$ such that $\chi(\stackrel{\circ}{E})=0$. Now although $\chi\left(\stackrel{\circ}{E}_{\ell}\right)=0$ for most exceptional varieties $E_{\ell}$ in a concrete example, we have that $\chi(\stackrel{\circ}{E})>0$ for a general configuration of curves on a projective plane or ruled surface $E^{0}$. So one could hope that only some simple configurations of curves $C_{i}, i \in T$, on $E^{0}$ satisfy $\chi(\stackrel{\circ}{E})=0$.

Projective planes. - Here the most simple configuration is the following.

EXAMPLE. - Let $E^{0} \cong \mathbb{P}^{2}$ and (after some permutation of the indices) $T=\{0, \ldots, k\}$ for $k \geq 2$. Let $C_{i}, 1 \leq i \leq k$, be projective lines all passing through the same point $P$ and $C_{0}$ a line not passing through $P$. Since $\chi\left(\mathbb{P}^{2}\right)=3$ and $\chi\left(\mathbb{P}^{1}\right)=2$ we have that $\chi(\stackrel{\circ}{E})=0$.

In Proposition 3.1 we (easily) prove that $\mathcal{R}=0$ in this case using the relations $(\mathrm{B} 1)$ and $(\mathrm{A})$.

Unfortunately there exists, after intensive examination, an amount of (essentially different) exotic configurations of curves $C_{i}$ on $E^{0} \cong \mathbb{P}^{2}$ such that $\chi(\stackrel{\circ}{E})=0$.

ExAmple. - Let $E^{0} \cong \mathbb{P}^{2}$ and (after some permutation of the indices) $T=\{0,1,2,3\}$. Let the $C_{i}$ be described in homogeneous coordinates $x, y, z$ by the equations

$$
\begin{aligned}
& C_{0}: y^{k} z=x^{k+1} \quad \text { where } \quad k \geq 2, \\
& C_{1}: y=0, \quad C_{2}: z=0, \quad C_{3}: x=0 .
\end{aligned}
$$

Since $\chi\left(C_{i}\right)=2$ for $0 \leq i \leq 3$ we have that $\chi(\stackrel{\circ}{E})=0$. 
In Proposition 3.5 we prove that $\mathcal{R}=0$ using relation (B1) and $2 k+2$ times relation (A), and the blowing-down technique of THEOREM 2.4.

In particular we succeeded in classifying all «low multiplicity» cases and then obtained by case distinction (THEOREM 4.9) :

Theorem. - If $\chi(\stackrel{\circ}{E})=0$ and $E^{0}$ is created by blowing-up a point of multiplicity at most 4 (on the strict transform of $f=0$ ), then $\mathcal{R}=0$.

In this context we would like to propose the following for eventual further investigations.

Conjecture. - Let $C_{i}, i \in T$, be irreducible curves in the complex projective plane $\mathbb{P}^{2}(\mathbb{C})$. If $\chi\left(\mathbb{P}^{2} \backslash \bigcup_{i \in T} C_{i}\right) \leq 0$ then all curves $C_{i}$ are rational.

Ruled Surfaces. - When $E^{0}$ is a ruled surface we especially studied the problem in the case that $E^{0}$ is created by blowing-up a curve $D$ at the stage of the resolution process where the strict transform of $f=0$ is already nonsingular. Even under this restriction a lot of different configurations of curves $C_{i}, i \in T$, occur on $E^{0}$ such that $\chi(\stackrel{\circ}{E})=0$.

ExAmple. - Let $E^{0}$ be a ruled surface $\Pi: E^{0} \rightarrow D$ over $D \cong \mathbb{P}^{1}$ and (after permutation of the indices) $T=\{1, \ldots, 5\}$. Let $C_{1}, C_{2}, C_{3}$ be sections and $C_{4}, C_{5}$ be fibers of $E^{0}$ intersecting as in figure 1 , where the intersection multiplicities of respectively $C_{1}$ and $C_{2}$ at $P$ and $C_{1}$ and $C_{3}$ at $Q$ are arbitrary positive numbers $m$ and $m^{\prime}$. Since $\chi\left(E^{0}\right)=4$ and all $C_{i} \cong \mathbb{P}^{1}$ we have that $\chi(\stackrel{\circ}{E})=0$.

By means of our blowing-down technique we prove in Proposition 5.6 simultaneously for all $m$ and $m^{\prime}$ that $\mathcal{R}=0$, using the relations (B1) and (B2) and $m+m^{\prime}$ times relation (A).

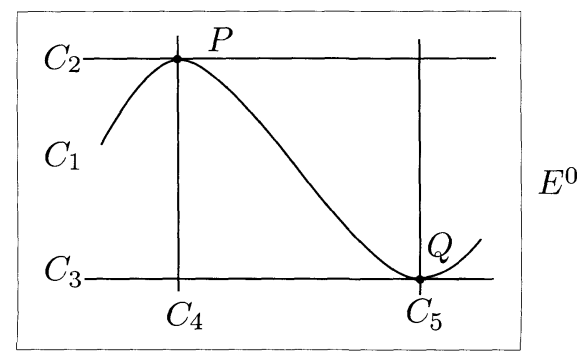

Figure 1

TOME $121-1993-\mathrm{N}^{\circ} 4$ 
We classified all cases where $D$ is a projective curve and finally obtained (TheOREM 5.9) :

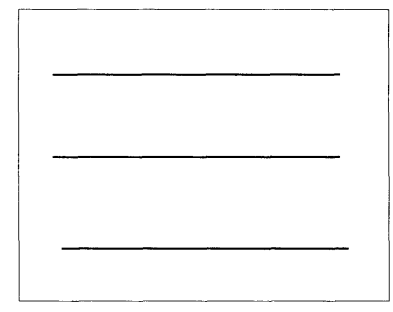

Figure 2

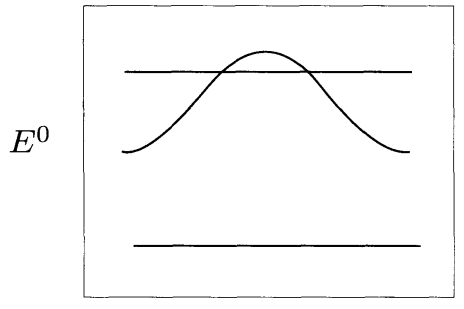

Figure 3

TheOrem. - Let $\chi(\stackrel{\circ}{E})=0$. Suppose that $E^{0}$ is created by blowing-up $a$ projective curve $D$ and that the strict transform of $f=0$ before this blowing-up is already nonsingular. Except for the two special situations when

(1) the genus of $D$ is 1 and the $C_{i}, i \in T$, consist of three nonintersecting sections of the ruled surface $E^{0}$ (Fig. 2), or

(2) $D \cong \mathbb{P}^{1}$ and the $C_{i}, i \in T$, consist of three sections intersecting as in figure 3 ,

we have that $\mathcal{R}=0$.

In the theorem above the second exceptional case is no obstruction for the monodromy conjecture (remark 5.7); and it is not clear whether the first can occur in a concrete situation (see remark 5.0). For example if $f=0$ has only absolutely isolated singularities this configuration is impossible (Proposition 5.12).

We return to the general case where $E^{0}$ is created at an arbitrary stage of the resolution process. If $E^{0}$ is a ruled surface over any projective curve of genus $g \geq 2$ we prove that only a few (simple) intersection configurations of the $C_{i}, i \in T$, can occur on $E^{0}$, and that $\mathcal{R}=0$ in these cases. The key result is (Proposition 5.13) :

Proposition. - Suppose that $E^{0}$ is a ruled surface over a projective curve of genus $g \geq 2$ and that at least two curves $C_{i}, i \in T$, are not fibers of $\Pi$. If $\chi(\stackrel{\circ}{E})=0$ then the $C_{i}, i \in T$, consist of a number of fibers and exactly two sections $C$ and $C^{\prime}$, such that each point of $C \cap C^{\prime}$ also belongs to some fiber $C_{i}$ (see Fig. 5.5).

Higher dimensions. - We also study some intersection configurations for arbitrary $n$. In particular if we suppose that $E^{0} \cong E^{m}(\cong E)$ we have the following results (Propositions 6.1 and 6.4). 
Proposition. - If $E \cong \mathbb{P}^{n}$ and the $C_{i}, i \in T$, consist of $k+1$ hyperplanes in general position $(1 \leq k \leq n)$, then $\chi(\stackrel{\circ}{E})=0$ and $\mathcal{R}=0$.

Proposition. - Suppose that $E$ is a projective space bundle $\Pi: E \rightarrow D$ over some nonsingular variety $D$ with fibers isomorphic to $\mathbb{P}^{1}$. Let $C_{i}$, $i \in T$, consist of two nonintersecting sections and of the inverse images $\Pi^{-1} B_{i}$ of nonsingular varieties $B_{i}$ in $D$ such that $\bigcup_{i} B_{i}$ has normal crossings on $D$ (see figure 6.4 ). Then $\chi(\stackrel{\circ}{E})=0$ and $\mathcal{R}=0$.

Terminology. - All schemes will be quasi-projective, a variety is an irreducible and reduced scheme, and (when not specified) points are assumed to be closed. The reduced scheme associated to a scheme $X$ is denoted by $X_{\text {red }}$.

Let $V$ be a subscheme of everywhere codimension one of a nonsingular scheme $X$. For all $x \in X$ we define the multiplicity of $x$ on $V$ as the maximal integer $m$, such that the $m$-th power of the maximal ideal of the local ring $\mathcal{O}_{X, x}$ of $x$ on $X$ contains the ideal of $V$ in $\mathcal{O}_{X, x}$.

\section{Relations between numerical data}

Let $k$ be an algebraically closed field of characteristic zero, let $f$ in $k\left[x_{1}, \ldots, x_{n+1}\right]$ be a non-constant polynomial, and let $Y$ denote the (reduced) zero set of $f$ in affine space $\mathbb{A}^{n+1}$ over $k$. We fix an embedded resolution $(X, h)$ for $Y$ in $\mathbb{A}^{n+1}$ in the sense of Hironaka's main theorem II [Hi, p. 142].

In particular $h: X \rightarrow \mathbb{A}^{n+1}$ is a finite succession of blowing-ups with nonsingular center, such that all points of the center have (the same) maximal multiplicity on the strict transform of $Y$. The restriction $h: X \backslash h^{-1} Y \rightarrow \mathbb{A}^{n+1} \backslash Y$ is an isomorphism, and $h^{-1} Y$ has only normal crossings in $X$.

Let $E_{i}, i \in S$, be the irreducible components of $\left(h^{-1} Y\right)_{\text {red }}$. These consist of the components $E_{i}, i \in I$, of the strict transform of $Y$, and of the exceptional divisors $E_{i}, i \in S \backslash I$, of the blowing-ups. The numerical data of the resolution $(X, h)$ for $Y$ are defined as follows. For each $E_{i}$, $i \in S$, let $N_{i}$ be the multiplicity of $E_{i}$ in the divisor of $f \circ h$ on $X$, and let $\left(\nu_{i}-1\right)$ be the multiplicity of $E_{i}$ in the divisor of $h^{*}\left(\mathrm{~d} x_{1} \wedge \cdots \wedge \mathrm{d} x_{n+1}\right)$ on $X$. We have that $N_{i}, \nu_{i} \in \mathbb{N}_{0}$; and if $f$ has no multiple components then all $E_{i}, i \in I$, have numerical data $\left(N_{i}, \nu_{i}\right)=(1,1)$.

Fix now one exceptional variety $E$ with numerical data $(N, \nu)$. There are basic relations (B1 and $\mathrm{B} 2$ ) associated to the creation of $E$ in the resolution process, and there are additional relations (A) associated to each blowing-up of the resolution that «changes» $E$.

TOME $121-1993-\mathrm{N}^{\circ} 4$ 
More precisely, the variety $E$ in the final resolution $X$ is in fact obtained by a finite succession of blowing-ups

$$
E^{0} \stackrel{\pi_{0}}{\longleftarrow} E^{1} \stackrel{\pi_{1}}{\longleftarrow} \cdots E^{i} \stackrel{\pi_{i}}{\longleftarrow} E^{i+1} \cdots \stackrel{\pi_{m-2}}{\longleftarrow} E^{m-1} \stackrel{\pi_{m-1}}{\longleftarrow} E^{m}=E
$$

with irreducible nonsingular center $\mathcal{D}_{i} \subset E^{i}$ and exceptional variety $C_{i+1} \subset E^{i+1}$ for $i=0, \ldots, m-1$. The variety $E^{0}$ is created at some stage of the global resolution process as the exceptional variety of a blowing-up with center $D$ and is isomorphic to a projective space bundle $\Pi: E^{0} \rightarrow D$ over $D$.

For $i=1, \ldots, m$ and for any variety $V \subset E^{j}, 0 \leq j<i$, let the repeated strict transform of $V$ in $E^{i}$ (by $\pi_{i-1} \circ \cdots \circ \pi_{j}$ ) be denoted by $V^{(i)}$. There are two kinds of intersections of $E$ with other components of $h^{-1} Y$. We have the repeated strict transforms $C_{1}^{(m)}, \ldots, C_{m}^{(m)}$ in $E$ of the exceptional varieties $C_{1}, \ldots, C_{m}$; and furthermore we have the repeated strict transforms $C_{i}^{(m)}$ in $E$ of varieties $C_{i}, i \in T$, (of codimension one) in $E^{0}$.

Those last varieties are the intersections of $E^{0}$ with previously created exceptional varieties in the global resolution process or with the strict transform of $Y$ (at the stage where $E^{0}$ is created). Remark that $\{1, \ldots, m\} \cap T=\emptyset$.

Theorem 0.1. - For each $i \in T \cup\{1, \ldots, m\}$ the variety $C_{i}^{(m)}$ is (an irreducible component of) the intersection of $E$ with exactly one other component of $\left(h^{-1} Y\right)_{\mathrm{red}}$. Let this component have numerical data $\left(N_{i}, \nu_{i}\right)$ and set $\alpha_{i}=\nu_{i}-(\nu / N) N_{i}$.

(1) Then we have

relation $(\mathrm{B} 1)$

$$
\sum_{i \in T} d_{i}\left(\alpha_{i}-1\right)+k=0
$$

where $k=n+1-\operatorname{dim} D$ and $d_{i}, i \in T$, is the degree of the intersection cycle $C_{i} \cdot F$ on $F$ for a general fiber $F \cong \mathbb{P}^{k-1}$ of $\Pi: E^{0} \rightarrow D$ over $a$ point of $D$. We also have

relation (B2) $\quad \sum_{\substack{i \in T \\ d_{i} \neq 0}} \frac{1}{k d_{i}^{k-1}}\left(\alpha_{i}-1\right) \Pi_{*}\left(C_{i}^{k}\right)+\sum_{\substack{i \in T \\ d_{i}=0}}\left(\alpha_{i}-1\right) B_{i}=K_{D}$

in Pic $D$, where $C_{i}^{k}$ is the $k$-th selfintersection of $C_{i}$ in $E^{0}, C_{i}=\Pi^{*} B_{i}$ when $d_{i}=0$, and $K_{D}$ is the canonical divisor on $D$. 
(2) Fix one $i \in\{0, \ldots, m-1\}$. Then we have relation $(\mathrm{A})$

$$
\alpha_{i+1}=\sum_{k \in T \cup\{1, \ldots, i\}} \mu_{k}\left(\alpha_{k}-1\right)+d
$$

where $\mu_{k}, k \in T \cup\{1, \ldots, i\}$, is the multiplicity of the generic point of $\mathcal{D}_{i}$ on $C_{k}^{(i)}$ and $d=\operatorname{codim}\left(\mathcal{D}_{i}, E^{i}\right)$.

Those relations are proved in respectively theorems $6.2,6.5$ and 4.4 of [V2].

ExAMPLE 0.2 . - If $D$ is a point we have that $E^{0}(\cong F) \cong \mathbb{P}^{n}$ and $d_{i}$ is just the degree of the hypersurface $C_{i}$ for $i \in T$. Moreover relation (B2) does not occur since Pic $D$ is trivial.

ExAmple 0.3. - When $n=1$ we have necessarily that $D$ is a point and moreover that $E^{0} \cong E$. So the relations (A) and (B2) do not occur and we easily see that relation $(\mathrm{B} 1)$ reduces to :

$$
\sum_{i \in T}\left(\alpha_{i}-1\right)+2=0
$$

ExAmPLE 0.4. - When $n=2$ we have that $D$ is a point or a nonsingular curve.

(1) If $D$ is a point then $E^{0} \cong \mathbb{P}^{2}$ and relation (B1) is

$$
\sum_{i \in T} d_{i}\left(\alpha_{i}-1\right)+3=0
$$

where $d_{i}$ is the degree of the curve $C_{i}$ in $E^{0}$.

(2) If $D$ is a nonsingular curve then $E^{0}$ is a ruled surface over $D$ and relation $(\mathrm{B} 1)$ is

$$
\sum_{i \in T} d_{i}\left(\alpha_{i}-1\right)+2=0
$$

where $d_{i}$ is the number of intersection points of the curve $C_{i}$ with a general fiber $F \cong \mathbb{P}^{1}$ of $\Pi: E^{0} \rightarrow D$. If moreover $D$ is a projective nonsingular curve then relation (B2) becomes a numerical relation by taking degrees in Pic $D$. More precisely if $g$ denotes the genus of $D$ and $\kappa_{i}$ the selfintersection number of $C_{i}$ in $E^{0}$, then we obtain

$$
\sum_{\substack{i \in T \\ d_{i} \neq 0}} \frac{\kappa_{i}}{2 d_{i}}\left(\alpha_{i}-1\right)+\sum_{\substack{i \in T \\ d_{i}=0}}\left(\alpha_{i}-1\right)=2 g-2 .
$$

Tоме $121-1993-\mathrm{N}^{\circ} 4$ 
The divisors $\Pi_{*}\left(C_{i}^{k}\right)$ occurring in relation (B2) are effectively computable in terms of concrete intersection cycles (see [V2, prop. 7.1]).

Example 0.5. - When $n=2$ and $D$ is a projective nonsingular curve we can describe the self-intersection numbers $\kappa_{i}$ in $(\ddagger)$ above as follows. Let $C_{0}, C_{1}, C_{2}$ occur with $d_{i} \neq 0$ for $i=0,1,2$. Denote $n_{12}=\operatorname{deg}\left(C_{1} \cdot C_{2}\right)$ and analogously $n_{01}$ and $n_{02}$. Then we have that

$$
\kappa_{0}=\frac{d_{0}}{d_{1} d_{2}}\left(d_{1} n_{02}+d_{2} n_{01}-d_{0} n_{12}\right) .
$$

In paragraph 1 we describe how to compute Igusa's local zeta function using an embedded resolution, and the impact of the monodromy conjecture on its poles. In the next sections we then compute the contribution $\mathcal{R}$ of a fixed exceptional variety in a number of cases when we expect it to be zero.

For surfaces we present in paragraph 2 the blowing-down technique that reduces the computation of a given $\mathcal{R}$ to an easy one. If the exceptional surface $E$ is created by blowing-up a point we study in paragraph 3 a series of general situations and in paragraph 4 all «low multiplicity $\rangle(\leq 4)$ cases. In paragraph 5 we treat the case that $E$ is created by blowing-up a curve. For arbitrary dimensions we give a number of applications in paragraph 6 .

\section{Igusa's local zeta function and the monodromy conjecture}

Let $K$ be a number field and $R$ its ring of algebraic integers. For any maximal ideal $\mathfrak{p}$ of $R$, let $R_{\mathfrak{p}}$ and $K_{\mathfrak{p}}$ denote the completion of respectively $R$ and $K$ with respect to the $\mathfrak{p}$-adic absolute value. Let $|x|$ denote this absolute value for $x \in K_{\mathfrak{p}}$, and let $q$ be the cardinality of the residue field $\bar{K}=R_{\mathfrak{p}} / \mathfrak{p} R_{\mathfrak{p}}$.

Definition 1.1. - Let $f(x) \in K[x], x=\left(x_{1}, \ldots, x_{n+1}\right)$. Then Igusa's local zeta function of $f$ is (the meromorphic continuation to $\mathbb{C}$ of)

$$
Z(s)=Z_{\mathfrak{p}}(s)=\int_{R_{\mathfrak{p}}^{n+1}}|f(x)|^{s}|\mathrm{~d} x|
$$

for $s \in \mathbb{C}, \operatorname{Re}(s)>0$, where $|\mathrm{d} x|$ denotes the Haar measure on $K_{\mathfrak{p}}^{n+1}$, normalized such that $R_{\mathfrak{p}}^{n+1}$ has measure one.

One can compute $Z(s)$ using an embedded resolution for $f=0$ in $\mathbb{A}^{n+1}\left(\mathbb{Q}^{a}\right)$, where $\mathbb{Q}^{a}$ is the algebraic closure of $\mathbb{Q}$ (and hence of $K$ ). Let $(X, h)$ be such a resolution, using now all notations of paragraph 0 . DENEF [D1, thm 2.4 and 3.1] proved the following formula. 
TheOREM 1.2. - Let $\bar{X}$ and $\bar{E}_{i}, i \in S$, denote the reduction modulo $\mathfrak{p} R_{\mathfrak{p}}$ of respectively $X$ and $E_{i}$. Then for almost all $\mathfrak{p}$ (i.e. for all except a finite number) we have

$$
Z(s)=q^{-(n+1)} \sum_{I \subset S} c_{I} \prod_{i \in I} \frac{q-1}{q^{\nu_{i}+s N_{i}}-1}
$$

where $c_{I}=\operatorname{card}\left\{a \in \bar{X} \mid a\right.$ is rational over $\bar{K}$, and $\left.a \in \bar{E}_{i} \Leftrightarrow i \in I\right\}$.

So all real poles of $Z(s)$ are part of the set $\left\{-\nu_{i} / N_{i} \mid i \in S\right\}$. These poles are connected with certain eigenvalues of monodromy by the following conjecture.

ConjeCture 1.3. - For all except a finite number of $\mathfrak{p}$ we have that, if $s$ is a pole of $Z_{\mathfrak{p}}(s)$, then $\mathrm{e}^{2 \pi i \operatorname{Re}(s)}$ is an eigenvalue of the monodromy acting on the cohomology (in some dimension) of the Milnor fiber of $f$ associated to some point of the hypersurface $f=0$.

Now by a formula of A'CAMPO [A, thm 3] we can connect also the eigenvalues of monodromy with the resolution $(X, h)$. For example if $f=0$ has just one isolated singular point $w$, then the monodromy in dimension $n$ associated to $w$ is the only non-trivial one and its characteristic polynomial $P(t)$ is $[\mathrm{A}$, thm 4$]$ :

$$
P(t)=\left[\frac{1}{t-1} \prod_{i \in S \backslash I}\left(t^{N_{i}}-1\right)^{\chi\left(\stackrel{\circ}{E}_{i}\right)}\right]^{(-1)^{n}}
$$

Here for $i \in S$ we set $\stackrel{\circ}{E}_{i}=E_{i} \backslash \bigcup_{j \neq i} E_{j}$, and for any scheme $V$ of finite type over $K$ we denote by $\chi(V)$ the Euler-Poincaré characteristic of $V(\mathbb{C})$ with respect to singular cohomology.

Combining the expression for $P(t)$ above with the monodromy conjecture we can generally expect that when $\chi\left(\stackrel{\circ}{E}_{j}\right)=0$ for an exceptional variety $E_{j}$, maybe even when $\chi\left(\stackrel{\circ}{E}_{j}\right) \leq 0$ for even $n$ or $\chi\left(\stackrel{\circ}{E}_{j}\right) \geq 0$ for odd $n$, then $E_{j}$ doesn't contribute to an eventual pole of $Z(s)$ in $-\nu_{j} / N_{j}$. Now it is striking and somewhat mysterious that in any concrete example we have that $\chi\left(\stackrel{\circ}{E}_{j}\right)=0$ for «most» exceptional varieties $E_{j}$.

In the next paragraphs we will verify this presumption for a lot of TOME $121-1993-\mathrm{N}^{\circ} 4$ 
different cases. For this purpose we now fix the following data :

- From now on we suppose that the number field $K$ is chosen large enough, that is the resolution $(X, h)$ over $\mathbb{Q}^{a}$ is entirely defined over $K$ itself.

- Fix an exceptional variety $E_{j}$ with $\chi\left(\stackrel{\circ}{E}_{j}\right) \leq 0$ for even $n$ $\left\{\begin{array}{l}\text { or } \chi\left(\stackrel{\circ}{E}_{j}\right) \geq 0 \text { for odd } n \text {. We suppose that } E_{j} \text { is } 《 \text { in general } \\ \text { position 》with respect to its numerical data, i.e. there is no } E_{i}, \\ i \in S \backslash\{j\}, \text { intersecting } E_{j} \text { with } \nu_{i} / N_{i}=\nu_{j} / N_{j} \text {. Denote by } \\ \mathcal{R}=\mathcal{R}_{\mathfrak{p}} \text { the contribution of } E_{j} \text { to the residue of the candidate- } \\ \text { pole }-\nu_{j} / N_{j} \text { for } Z_{\mathfrak{p}}(s) .\end{array}\right.$

(Eventually there can be other contributions to this residue but those don't interfere with $\mathcal{R}$ because of the «general position» condition.) We will give examples of this situation, mostly for surfaces, and prove that $\mathcal{R}=0$ (for almost all $\mathfrak{p}$ ).

Note that $\mathcal{R}$ is an expression in the $\alpha_{i}=\nu_{i}-\left(\nu_{j} / N_{j}\right) N_{i}(\neq 0$ !) for intersecting $E_{i}$, so we can use the relations (A) and (B) between numerical data of paragraph 0 to prove that $\mathcal{R}=0$.

REMARK 1.4.

(i) In fact the result that $\mathcal{R}=0$ in the data $\left(^{*}\right)$ is in general not implied by the monodromy conjecture. It is implied in the special case that $f=0$ has only isolated singularities when there is $n o E_{i}, i \in S \backslash\{j\}$, with $N^{\prime} \mid N_{i}$, where $\nu_{j} / N_{j}=\nu^{\prime} / N^{\prime}$ and $\nu^{\prime}$ and $N^{\prime}$ are coprime. (See also remark 5.7 (b).)

(ii) When $\chi\left(\stackrel{\circ}{E}_{j}\right)>0$ for even $n$ or $\chi\left(\stackrel{\circ}{E}_{j}\right)<0$ for odd $n$ we have usually that $\mathcal{R} \neq 0$.

We now describe the only two possible examples for curves $(n=1)$, illustrating the general idea. See also Strauss [S, thm 2] and (in a more general setting) Meuser [Me1, thm 1] and Igusa [Ig3, thm 1] for absolutely analytically irreducible $f\left(x_{1}, x_{2}\right)$, and LoESER [L1, lemme IV.2.3] for arbitrary $f\left(x_{1}, x_{2}\right)$.

Proposition 1.5. - For $n=1$ we have that $\chi\left(\stackrel{\circ}{E}_{j}\right) \geq 0$ if and only if $E_{j}$ intersects one or two times another curve $E_{i}, i \in S$, and then $\chi\left(\stackrel{\circ}{E}_{j}\right)$ is respectively 1 and 0 . In both cases we have that $\mathcal{R}=0$.

Proof. - Since $E_{j} \cong \mathbb{P}^{1}$ the claims about $\chi\left(\stackrel{\circ}{E}_{j}\right)$ are evident. Say $E_{j}$ intersects $E_{1}$ and $E_{2}$ (see figure 1.1 next page ). Then the contribution 


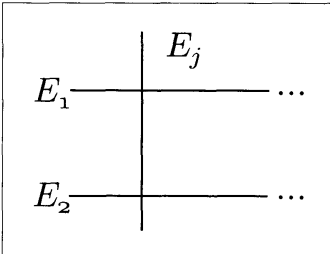

Figure 1.1

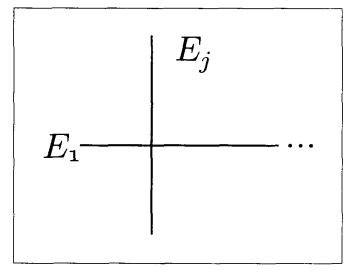

Figure 1.2

of $E_{j}$ to $Z(s)$ consists of

$$
\begin{aligned}
\frac{1}{q^{2}}\left[(q-1) \frac{q-1}{q^{\nu_{j}+s N_{j}}-1}+1 \cdot \frac{(q-1)^{2}}{\left(q^{\nu_{j}+s N_{j}}-1\right)\left(q^{\nu_{1}+s N_{1}}-1\right)}\right. \\
\left.+1 \cdot \frac{(q-1)^{2}}{\left(q^{\nu_{j}+s N_{j}}-1\right)\left(q^{\nu_{2}+s N_{2}}-1\right)}\right]
\end{aligned}
$$

and we thus have that

$$
\mathcal{R}=(q-1)\left(1+\frac{1}{q^{\alpha_{1}}-1}+\frac{1}{q^{\alpha_{2}}-1}\right)=\frac{(q-1)\left(q^{\alpha_{1}+\alpha_{2}}-1\right)}{\left(q^{\alpha_{1}}-1\right)\left(q^{\alpha_{2}}-1\right)}
$$

Now $\alpha_{1}+\alpha_{2}=0$ (by example 0.3 ) and so $\mathcal{R}=0$. If $E_{j}$ intersects only $E_{1}$ (Fig. 1.2) then

$$
\mathcal{R}=q+\frac{q-1}{q^{\alpha_{1}}-1}=\frac{q^{\alpha_{1}+1}-1}{q^{\alpha_{1}}-1}=0
$$

since $\alpha_{1}+1=0$.

REMARK 1.6. - The exact contribution of $E_{j}$ to the residue of $-\nu_{j} / N_{j}$ for $Z(s)$ in the proof above is :

$$
\frac{q-1}{q^{2} N_{j}(\log q)} \mathcal{R}
$$

We will always neglect such constant factors. 


\section{Techniques and notations}

From now on until paragraph 6 we suppose that $n=2$.

Remember that $E_{j}$ in the data $\left(^{*}\right)$ is obtained by a finite succession of blowing-ups

$$
E^{0} \stackrel{\pi_{0}}{\longrightarrow} E^{1} \stackrel{\pi_{1}}{\longrightarrow} \cdots E^{i} \stackrel{\pi_{i}}{\longrightarrow} E^{i+1} \cdots \stackrel{\pi_{m-2}}{\longrightarrow} E^{m-1} \stackrel{\pi_{m-1}}{\longrightarrow} E^{m}=E_{j}
$$

with a point as center and an exceptional curve $C_{i}\left(\cong \mathbb{P}^{1}\right) \subset E^{i}$ for $i=1, \ldots, m$. The surface $E^{0}$ is created at some stage of the global resolution $h$ as the exceptional variety of a blowing-up with center $D$, and the $E^{i}, 1 \leq i \leq m$, are its strict transforms by the next blowing-ups of the global resolution process.

The variety $D$ can be a point or a nonsingular curve; in the first case $E^{0} \cong \mathbb{P}^{2}$ and in the latter $E^{0}$ is a ruled surface over $D$. Let $C_{i}$, $i \in T$, denote the (reduced) intersections of $E^{0}$ with other exceptional surfaces or with the strict transform of $Y$. Then for all $i=1, \ldots, m$ the (reduced) intersections of $E^{i}$ with other exceptional varieties or with the strict transform of $Y$ are the curves $C_{\ell}, \ell \in T \cup\{1, \ldots, i\}$.

To avoid complicated indices we use the same notations for the curves $C_{\ell}$ and their strict transform in the $E^{i}$.

We also have that $\bigcup_{i \in T \cup\{1, \ldots, m\}} C_{i}$ has normal crossings in $E^{m}$. And by Theorem 0.1 we can associate to the $C_{i}, i \in T \cup\{1, \ldots, m\}$, a pair of numerical data $\left(N_{i}, \nu_{i}\right)$ such that the numbers $\alpha_{i}=\nu_{i}-\left(\nu_{j} / N_{j}\right) N_{i}$ occur in $\mathcal{R}$.

\section{Notation 2.1}

(i) We can define $\stackrel{\circ}{E}^{i}$ as in paragraph 1 for $i=0, \ldots, m$; then the $\chi\left(\stackrel{\circ}{E}^{i}\right)$ are all equal and we will denote this number as $\chi(\stackrel{\circ}{E})$.

(ii) For any scheme $V$ defined over $\bar{K}$ we set :

$$
\operatorname{card} V=\operatorname{card}\{a \in V \mid a \text { is rational over } \bar{K}\} \text {. }
$$

We now explain a technique to reduce a given situation with data $\left(^{*}\right)$ to a situation where we can easily prove that $\mathcal{R}=0$. Therefore we suppose that all schemes (and their intersections) in the next statements are defined over $\bar{K}$.

Definition 2.2. - Let $V$ be a nonsingular surface and let $C_{i}, i \in T$, be irreducible curves on $V$ such that $\bigcup_{i \in T} C_{i}$ has normal crossings on $V$. To 
each $C_{i}, i \in T$, we associate a number $\alpha_{i} \in \mathbb{Q}_{0}$. Denote $\stackrel{\circ}{V}=V \backslash \bigcup_{i \in T} C_{i}$ and $\stackrel{\circ}{C}_{i}=C_{i} \backslash \bigcup_{\ell \neq i} C_{\ell}$. Then we define :

$$
\begin{aligned}
\mathcal{R}\left(V, \bigcup_{i \in T} C_{i}\right)=\operatorname{card} \stackrel{\circ}{V} & +(q-1) \sum_{i \in T} \frac{\operatorname{card} \stackrel{\circ}{C}_{i}}{q^{\alpha_{i}}-1} \\
& +(q-1)^{2} \sum_{\substack{\{i, j\} \subset T \\
i \neq j}} \frac{\operatorname{card}\left(C_{i} \cap C_{j}\right)}{\left(q^{\alpha_{i}}-1\right)\left(q^{\alpha_{j}}-1\right)} .
\end{aligned}
$$

Of course the $\mathcal{R}$ in $\left(^{*}\right)$ is equal to $\mathcal{R}\left(E^{m}, \bigcup_{i \in T \cup\{1, \ldots, m\}} C_{i}\right)$.

Proposition 2.3. - Let $V$ be a nonsingular surface and $C_{i}, i \in T$, irreducible curves on $V$ with associated number $\alpha_{i} \in \mathbb{Q}_{0}$ such that $\bigcup_{i \in T} C_{i}$ has normal crossings on $V$. Let $g: \widetilde{V} \rightarrow V$ be the blowing-up with center a point $P$ of $V$ and exceptional curve $C$ with associated number $\alpha=\sum_{i \in T, P \in C_{i}}\left(\alpha_{i}-1\right)+2 \neq 0$. (Denote the strict transform of $C_{i}, i \in T$, in $\widetilde{V}$ also by $C_{i}$.) Then $\left(\bigcup_{i \in T} C_{i}\right) \cup C$ has normal crossings on $\widetilde{V}$ and

$$
\mathcal{R}\left(\tilde{V},\left(\bigcup_{i \in T} C_{i}\right) \cup C\right)=\mathcal{R}\left(V, \bigcup_{i \in T} C_{i}\right) .
$$
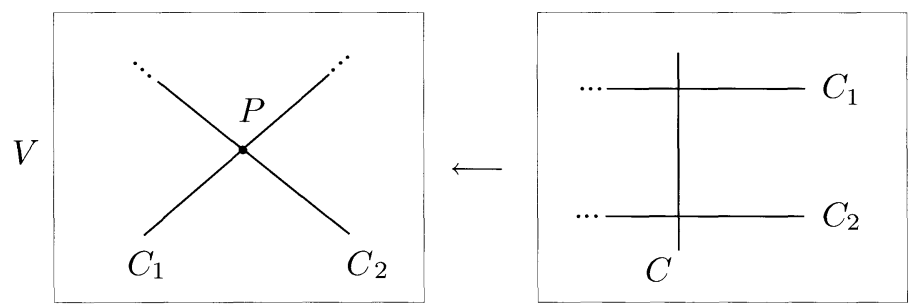

Figure 2.1

Proof. - The point $P$ can belong to zero, one or two curves $C_{i}, i \in T$. Suppose that $P \in C_{1} \cap C_{2}$ (Fig. 2.1). (The other two cases can be proved analogously.)

By definition the contributions to

$$
\mathcal{R}\left(V, \bigcup_{i \in T} C_{i}\right) \text { and } \mathcal{R}\left(\widetilde{V},\left(\bigcup_{i \in T} C_{i}\right) \cup C\right)
$$

are the same except for the terms

$$
\frac{(q-1)^{2}}{\left(q^{\alpha_{1}}-1\right)\left(q^{\alpha_{2}}-1\right)}
$$

TOME $121-1993-\mathrm{N}^{\circ} 4$ 
for $\mathcal{R}\left(V, \bigcup_{i \in T} C_{i}\right)$ and

$$
(q-1) \frac{q-1}{q^{\alpha}-1}+\frac{(q-1)^{2}}{\left(q^{\alpha}-1\right)\left(q^{\alpha_{1}}-1\right)}+\frac{(q-1)^{2}}{\left(q^{\alpha}-1\right)\left(q^{\alpha_{2}}-1\right)}
$$

for $\mathcal{R}\left(\widetilde{V},\left(\bigcup_{i \in T} C_{i}\right) \cup C\right)$. But since $\alpha=\alpha_{1}+\alpha_{2}$ also those terms are equal.

So by Proposition 2.3 and relation (A) we can treat the different situations in the data $\left(^{*}\right)$ by determining the different situations with $\chi(\stackrel{\circ}{E}) \leq 0$, where $E^{0} \cong \mathbb{P}^{2}$ or $E^{0}$ is a ruled surface, and by studying $\mathcal{R}\left(E^{m}, \bigcup_{i \in T \cup\{1, \ldots, m\}} C_{i}\right)$, where we now may suppose that $\bigcup_{i \in T \cup\{1, \ldots, m\}} C_{i}$ in $E^{m}$ is the canonical embedded resolution of $\bigcup_{i \in T} C_{i}$ in $E^{0}$.

Moreover the following theorem will simplify the computations enormously.

TheOREm 2.4. - Let $\widetilde{V}$ be a nonsingular surface and $C$ and $C_{i}, i \in T$, irreducible curves (with associated numbers $\alpha$ and $\alpha_{i} \in \mathbb{Q}_{0}$ ) such that $\left(\bigcup_{i \in T} C_{i}\right) \cup C$ has normal crossings on $\tilde{V}$. Let also

$$
K=(\alpha-1) C+\sum_{i \in T}\left(\alpha_{i}-1\right) C_{i}
$$

be the canonical divisor on $\tilde{V}$. Suppose that $C \cong \mathbb{P}^{1}$ and that $C$ intersects one or two times a curve $C_{i}, i \in T$.

(i) If $C$ intersects either $C_{1}$ and $C_{2}$ with $\alpha=\alpha_{1}+\alpha_{2}$, or only $C_{1}$ with $\alpha=\alpha_{1}+1$, then $\widetilde{V}$ can be considered as the blown-up of a nonsingular surface $V$ (with exceptional curve $C$ ).

(ii) Moreover $\bigcup_{i \in T} C_{i}$ has normal crossings on $V$ and

$$
\mathcal{R}\left(\tilde{V},\left(\bigcup_{i \in T} C_{i}\right) \cup C\right)=\mathcal{R}\left(V, \bigcup_{i \in T} C_{i}\right) .
$$

Proof.

(i) By Castelnuovo's criterion [Ha, V, thm 5.7] we only have to show that $\kappa=-1$, where $\kappa$ is the self-intersection number of $C$. If $C$ intersects $C_{1}$ and $C_{2}$ (Fig. 2.1) then the adjunction formula [Ha, V, prop. 1.5] for $C$ on $\widetilde{V}$ yields :

$$
-2=C \cdot(C+K)=C \cdot\left[\alpha C+\sum_{i \in T}\left(\alpha_{i}-1\right) C_{i}\right]=\kappa \alpha+\alpha_{1}+\alpha_{2}-2 .
$$

So we have that $\kappa=-1 \Leftrightarrow \alpha=\alpha_{1}+\alpha_{2}$. The other case is analogous.

(ii) This is immediate by Proposition 2.3. 
Thus starting from $\bigcup_{i \in T \cup\{1, \ldots, m\}} C_{i}$ in $E^{m}$ we can (by [V2, prop. 2.1 and lemma 4.1]) blow down every time a curve $C_{i}$ occurs that intersects either just two curves $C_{1}$ and $C_{2}$ with $\alpha_{i}=\alpha_{1}+\alpha_{2}$, or just one curve $C_{1}$ with $\alpha_{i}=\alpha_{1}+1$. Say we finally obtain the surface $Z$ with $\bigcup_{i \in T^{\prime}} C_{i}$ occurring on $Z$; then :

$$
\mathcal{R}\left(E^{m}, \bigcup_{i \in T \cup\{1, \ldots, m\}} C_{i}\right)=\mathcal{R}\left(Z, \bigcup_{i \in T^{\prime}} C_{i}\right)
$$

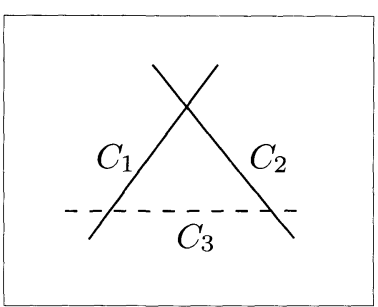

Figure 2.2

Now except for the almost trivial case that $E^{0} \cong E^{m} \cong \mathbb{P}^{2}$, where $\chi(\stackrel{\circ}{E}) \leq 0$ if and only if the $C_{i}, i \in T$, consist of two or three lines in general position (Fig. 2.2), we will succeed for all treated examples in reducing $E^{m}$ by Theorem 2.4 to a surface $Z$ that satisfies the conditions of Proposition 2.5 below.

More precisely if $E^{0} \cong \mathbb{P}^{2}$ or $E^{0}$ is a ruled surface we will blow down $E^{m}$ respectively $(m-1)$ and $m$ times.

Proposition 2.5. - Let $Z$ be a nonsingular surface and let $\bigcup_{i \in T^{\prime}} C_{i}$ (with associated numbers $\alpha_{i} \in \mathbb{Q}_{0}$ ) have normal crossings on $Z$. Suppose that $C_{i} \cong \mathbb{P}^{1}$ for $i=1, \ldots, k$ (where $\left.k \geq 0\right)$. Let either :

(i) $T^{\prime}=\{1, \ldots, k+2\}$, where $C_{k+1} \cong C_{k+2}$ and $C_{k+1} \cap C_{k+2}=\emptyset$, and with $\alpha_{k+1}+\alpha_{k+2}=0$, or

(ii) $T^{\prime}=\{1, \ldots, k+1\}$ with $\alpha_{k+1}=-1$.

Let the curves $C_{i}, i \in T^{\prime}$, intersect as given by figure 2.3 and suppose in both cases also that $\operatorname{card} Z=(q+1) \operatorname{card} C_{k+1}=(q+1) \operatorname{card} C_{k+2}$. Then $\mathcal{R}\left(Z, \bigcup_{i \in T^{\prime}} C_{i}\right)=0$.

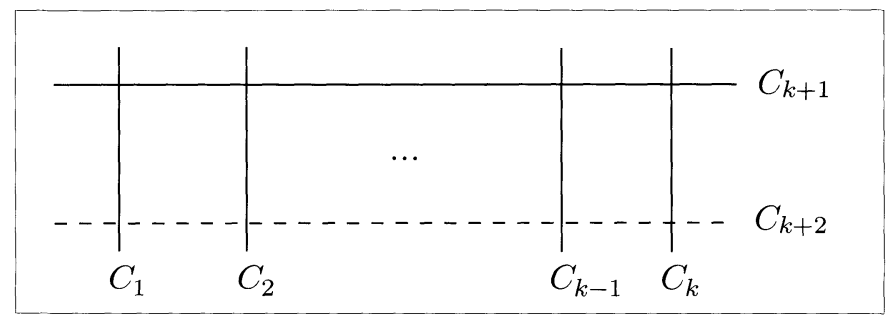

Figure 2.3

TOME $121-1993-\mathrm{N}^{\circ} 4$ 
Proof.

(i) Denote $n_{q}=\operatorname{card} C_{k+1}$. Then :

$$
\begin{aligned}
& \mathcal{R}\left(Z, \bigcup_{i \in T^{\prime}} C_{i}\right) \\
& =(q-1)^{2} \sum_{i=1}^{k} \frac{1}{q^{\alpha_{i}}-1}\left(\frac{1}{q^{\alpha_{k+1}}-1}+\frac{1}{q^{\alpha_{k+2}}-1}\right) \\
& \quad+(q-1) \sum_{i=1}^{k} \frac{q-1}{q^{\alpha_{i}}-1} \\
& \quad+(q-1)\left(\frac{n_{q}-k}{q^{\alpha_{k+1}}-1}+\frac{n_{q}-k}{q^{\alpha_{k+2}}-1}\right)+(q-1)\left(n_{q}-k\right) \\
& =(q-1) \frac{\left(q^{\alpha_{k+1}+\alpha_{k+2}}-1\right)}{\left(q^{\alpha_{k+1}}-1\right)\left(q^{\alpha_{k+2}}-1\right)}\left\{n_{q}-k+(q-1) \sum_{i=1}^{k} \frac{1}{q^{\alpha_{i}}-1}\right\} \\
& =0 .
\end{aligned}
$$

Case (ii) is analogous.

Actually in most examples $E^{0}$ will be a rational surface, and then we will use Proposition 2.5 with $C_{k+1}\left(\cong C_{k+2}\right) \cong \mathbb{P}^{1}$ and card $Z=(q+1)^{2}$. Finally we mention the following technique.

Proposition 2.6. - Let $\mathcal{R}_{1}$ denote the contribution (as in the data $\left(^{*}\right)$ ) associated to a certain configuration of $\left(\bigcup_{i \in T^{\prime}} C_{i}\right) \cup C$ on $E^{0}$ (with $\chi(\stackrel{\circ}{E}) \leq 0)$, and let $\mathcal{R}_{2}$ denote the contribution associated to the configuration of $\bigcup_{i \in T^{\prime}} C_{i}$ on $E^{0}$ (with $\chi(\stackrel{\circ}{E}) \leq 0$ ) obtained by just dropping $C$. If $\mathcal{R}_{2}$ is obtained from $\mathcal{R}_{1}$ by substituting $\alpha=1$ and if $\alpha$ is not necessarily different from 1 in $\mathcal{R}_{1}$, then $\mathcal{R}_{1}=0$ implies $\mathcal{R}_{2}=0$.

In concrete examples we often encounter this substitution property if the resolution process is essentially the same for the two configurations, for then the relations (B) and (A) for the second are obtained from the same relations for the first by substituting $\alpha=1$. For example in Proposition 2.5 we have that case (ii) is implied by case (i) using Proposition 2.6.

\section{Starting with a projective plane}

We suppose now that $E^{0} \cong \mathbb{P}^{2}$.

We consider in this section a series of general situations with $\chi(\stackrel{\circ}{E}) \leq 0$ and where the $C_{i}, i \in T$, consist of either lines, or curves of degree $k+1$ 
with a singular point of multiplicity $k$. Then in the next section we classify all cases with $\chi(\stackrel{\circ}{E}) \leq 0$ occurring when the point $D$ has multiplicity at most 4 on the strict transform of $Y$.

REMARK 3.0.

(i) We use homogeneous coordinates $(x, y, z)$ in $E^{0} \cong \mathbb{P}^{2}$, and affine coordinates $(x, y)$ in the affine chart $z \neq 0$. We will also use primes $\left(C^{\prime}\right)$ and double primes $\left(C^{\prime \prime}\right)$ to describe the $C_{i}, i \in T \cup\{1, \ldots, m\}$.

(ii) To describe configurations of the $C_{i}, i \in T$, we use the notion of equivalence between (reduced) curves in a nonsingular surface of [Ha, V, rem. 3.9.4]. Roughly two curves are equivalent if they have the same (canonical) embedded resolution graph.

(iii) We can find concrete examples of every possible configuration of curves $C_{i}, i \in T$, on $E^{0}$. When $C_{i}$ is given by the homogeneous equation $f_{i}(x, y, z)=0$ of degree $d_{i}$, take for instance a surface $Y$ in $\mathbb{A}^{3}$ with an isolated singularity in the origin given by

$$
f\left(x_{1}, x_{2}, x_{3}\right)=\prod_{i \in T} f_{i}\left(x_{1}, x_{2}, x_{3}\right)+\left[\text { terms of degree at least } 1+\sum_{i \in T} d_{i}\right]
$$

(see also Proposition 4.0).

Proposition 3.1. - Let $k \geq 2$ and let $C_{i}, 1 \leq i \leq k$, be projective lines all passing through the same point P. Let either (Fig. 3.1)

(i) card $T=k+1$, where $C_{0}$ is a line not passing through $P$, so $\chi(\stackrel{\circ}{E})=0$, or

(ii) $\operatorname{card} T=k$, so $\chi(\stackrel{\circ}{E})=2-k$.

Then $\mathcal{R}=0$.

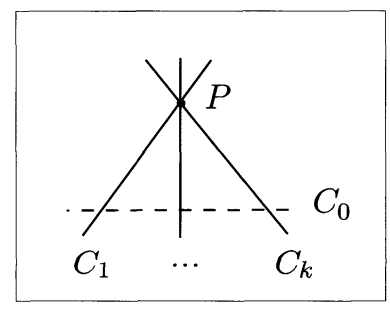

Figure 3.1

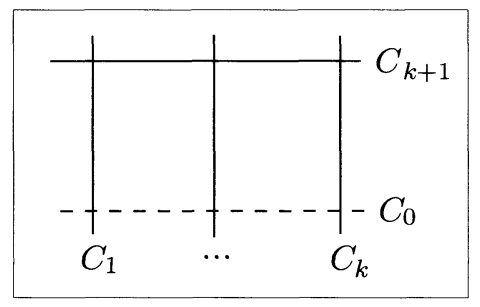

Figure 3.2

TOME $121-1993-\mathrm{N}^{\circ} 4$ 
Proof.

(i) If $k \geq 3$ we obtain the canonical embedded resolution of $\bigcup_{0 \leq i \leq k} C_{i}$ by constructing one blowing-up with center $P$ and exceptional variety $C_{k+1}$ (Fig. 3.2). The relations (B1) and (A) yield respectively

$$
\sum_{i=0}^{k}\left(\alpha_{i}-1\right)+3=0 \quad \text { and } \quad \alpha_{k+1}=\sum_{i=1}^{k}\left(\alpha_{i}-1\right)+2
$$

and imply that $\alpha_{k+1}+\alpha_{0}=0$. Using Proposition 2.5 we conclude that $\mathcal{R}=0$. If $k=2$ one can compute easily that $\mathcal{R}=0$ using relation (B1).

(ii) We can derive this case from (i) by Proposition 2.6 and the following observation. By Theorem 1.2 we have for case (i) that

$$
\begin{gathered}
\mathcal{R}=(q-1)^{2} \sum_{i=1}^{k} \frac{1}{q^{\alpha_{i}}-1}\left(\frac{1}{q^{\alpha_{k+1}}-1}+\frac{1}{q^{\alpha_{0}}-1}\right)+(q-1) \sum_{i=1}^{k} \frac{q-1}{q^{\alpha_{i}}-1} \\
+(q-1)\left(\frac{q+1-k}{q^{\alpha_{k+1}}-1}+\frac{q+1-k}{q^{\alpha_{0}}-1}\right)+(q-1)(q+1-k)
\end{gathered}
$$

and for case (ii) that

$$
\begin{aligned}
\mathcal{R}=\frac{(q-1)^{2}}{q^{\alpha_{k+1}}-1} \sum_{i=1}^{k} \frac{1}{q^{\alpha_{i}}-1}+( & -1) \sum_{i=1}^{k} \frac{q}{q^{\alpha_{i}}-1} \\
& +\frac{(q-1)(q+1-k)}{q^{\alpha_{k+1}}-1}+q(q+1-k),
\end{aligned}
$$

which is clearly obtained from the previous $\mathcal{R}$ by substituting $\alpha_{0}=1$.

Proposition 3.2. - Let $\operatorname{card} T=1+s+t$ where $s \geq 1$ and $t \geq 0$. Let $\bigcup_{i \in T} C_{i}$ be equivalent to $\left(\bigcup_{0 \leq i \leq s} C_{i}\right) \cup\left(\bigcup_{1 \leq j \leq t} C_{j}^{\prime}\right)$ with equations

$$
C_{0}: \prod_{i=1}^{s}\left(y-a_{i} x\right)^{m_{i}}=x^{k+1}
$$

where $m_{i} \geq 1$ for $i=1, \ldots, s$ and $k=\sum_{i=1}^{s} m_{i}(\geq 2)$,

$$
\begin{array}{lll}
C_{i}: y=a_{i} x & \text { for } & i=1, \ldots, s \\
C_{j}^{\prime}: y=b_{j} x & \text { for } & j=1, \ldots, t
\end{array}
$$

where the numbers $a_{i}$ and $b_{j}$ are all different (see figure 3.3 next page). So $\chi(\stackrel{\circ}{E})=0$. Then $\mathcal{R}=0$. 


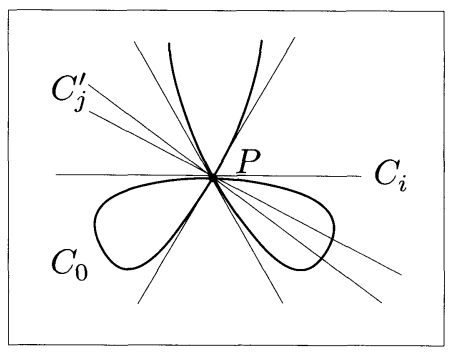

Figure 3.3

Proof. - Let $C^{\prime}$ denote the exceptional curve of the blowing-up with center $P$ (Fig. 3.4).

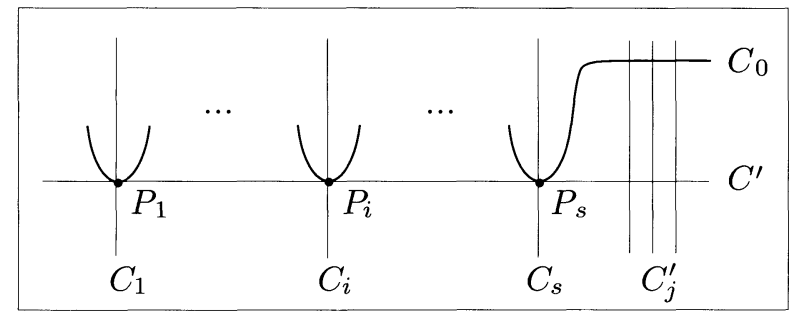

Figure 3.4

Since for $i=1, \ldots, s$ the intersection multiplicity of the (nonsingular) curves $C_{0}$ and $C^{\prime}$ at $P_{i}$ is $m_{i}$, we obtain an embedded resolution of $\bigcup_{i \in T} C_{i}$ by constructing for all $i=1, \ldots, s$ a series of $m_{i}$ blowing-ups with exceptional curves $C_{i 1}, C_{i 2}, \ldots, C_{i m_{i}}$ which intersect as given by figure 3.5 .

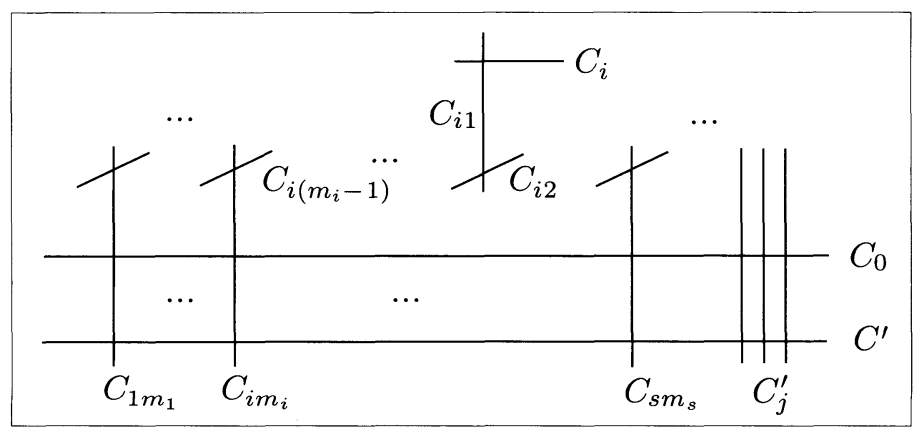

Figure 3.5

TOME $121-1993-\mathrm{N}^{\circ} 4$ 
Relation (B1) and relation (A) associated to $C^{\prime}$ yield respectively

$$
\begin{aligned}
& (k+1)\left(\alpha_{0}-1\right)+\sum_{i=1}^{s}\left(\alpha_{i}-1\right)+\sum_{j=1}^{t}\left(\alpha_{j}^{\prime}-1\right)+3=0 \\
& \alpha^{\prime}=k\left(\alpha_{0}-1\right)+\sum_{i=1}^{s}\left(\alpha_{i}-1\right)+\sum_{j=1}^{t}\left(\alpha_{j}^{\prime}-1\right)+2
\end{aligned}
$$

implying that

$$
\alpha_{0}+\alpha^{\prime}=0
$$

For all $i=1, \ldots, s$ we have by relation (A) also

$$
\left\{\begin{array}{l}
\alpha_{i 1}=\alpha_{0}+\alpha^{\prime}+\alpha_{i}-1 \\
\alpha_{i 2}=\alpha_{0}+\alpha^{\prime}+\alpha_{i 1}-1 \\
\alpha_{i 3}=\alpha_{0}+\alpha^{\prime}+\alpha_{i 2}-1 \\
\quad \cdots \\
\alpha_{i\left(m_{i}-1\right)}=\alpha_{0}+\alpha^{\prime}+\alpha_{i\left(m_{i}-2\right)}-1 \\
\alpha_{i m_{i}}=\alpha_{0}+\alpha^{\prime}+\alpha_{i\left(m_{i}-1\right)}-1
\end{array}\right.
$$

and thus

$$
\left\{\begin{array}{l}
\alpha_{i}=\alpha_{i 1}+1 \\
\alpha_{i 1}=\alpha_{i 2}+1 \\
\alpha_{i 2}=\alpha_{i 3}+1 \\
\quad \cdots \\
\alpha_{i\left(m_{i}-2\right)}=\alpha_{i\left(m_{i}-1\right)}+1 \\
\alpha_{i\left(m_{i}-1\right)}=\alpha_{i m_{i}}+1
\end{array}\right.
$$

Using (5) we can blow down $\sum_{i=1}^{s} m_{i}=k$ times following the idea of TheOrem 2.4 to obtain the situation as described by figure 3.6 next page. By (3) and Proposition 2.5 we conclude that $\mathcal{R}=0$. (Since we have blown-up $k+1$ times and blown-down only $k$ times the cardinality of the final surface over $\bar{K}$ is indeed $(q+1)^{2}$.)

REMARK. - In order to appreciate the blowing-down technique we state the terrifying expression for the $\mathcal{R}$ of Proposition 3.2, given 


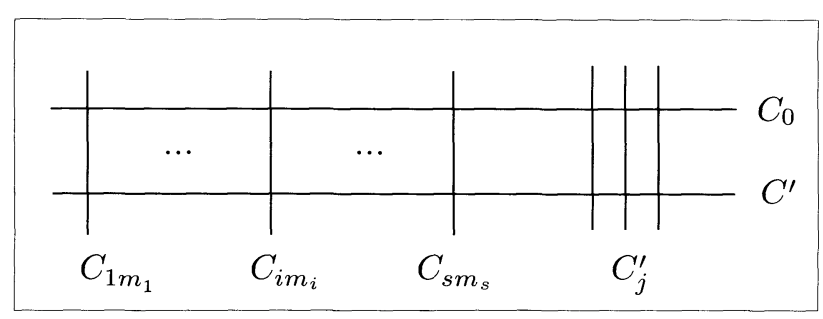

Figure 3.6

by THEOREM 1.2 :

$$
\begin{aligned}
\mathcal{R} & =(q-1)\left(\frac{q+1-s-t}{q^{\alpha_{0}}-1}+\frac{q+1-s-t}{q^{\alpha^{\prime}}-1}\right) \\
& +(q-1) \sum_{j=1}^{t} \frac{q-1}{q^{\alpha_{j}^{\prime}}-1}+(q-1)^{2} \sum_{j=1}^{t} \frac{1}{q^{\alpha_{j}^{\prime}}-1}\left(\frac{1}{q^{\alpha_{0}}-1}+\frac{1}{q^{\alpha^{\prime}}-1}\right) \\
& +(q-1) \sum_{i=1}^{s} \frac{q-2}{q^{\alpha_{i m_{i}}}-1}+(q-1)^{2} \sum_{i=1}^{s} \frac{1}{q^{\alpha_{i m_{i}}-1}}\left(\frac{1}{q^{\alpha_{0}}-1}+\frac{1}{q^{\alpha^{\prime}}-1}\right) \\
& +\sum_{i=1}^{s}\left[\frac{(q-1) q}{q^{\alpha_{i}}-1}+\frac{(q-1)^{2}}{\left(q^{\alpha_{i}}-1\right)\left(q^{\alpha_{i 1}}-1\right)}\right] \\
& +\sum_{i=1}^{s}\left[\sum_{\ell=1}^{m_{i}-1} \frac{(q-1)^{2}}{q^{\alpha_{i \ell}}-1}+\sum_{\ell=1}^{m_{i}-1} \frac{(q-1)^{2}}{\left(q^{\alpha_{i \ell}}-1\right)\left(q^{\alpha_{i(\ell+1)}}-1\right)}\right] \\
& +\left[q^{2}+q+1-(q+1-s)-s q-t(q-1)-1\right] .
\end{aligned}
$$

Trying to prove that $\mathcal{R}=0$, starting from this expression, is not funny at all.

Using the same techniques as in the proof of Proposition 3.2 but with more computations, we can also prove the two following cases.

Proposition 3.3. - Let $\operatorname{card} T=s+2$ where $s \geq 2$. Let $\bigcup_{i \in T} C_{i}$ be equivalent to $\left(\bigcup_{1 \leq i \leq s} C_{i}\right) \cup C \cup C^{\prime}$ with equations

$$
C_{i}: y^{m} x^{k-m}=x^{k+1}+a_{i} y^{k+1} \quad \text { for } \quad i=1, \ldots, s
$$

where $k \geq 2,1 \leq m \leq k-1$, and the $a_{i}$ are all different and not equal to zero,

$$
C: x=0 \quad \text { and } \quad C^{\prime}: y=0
$$

(Fig. 3.7 next page). So $\chi(\stackrel{\circ}{E})=0$. Then $\mathcal{R}=0$. 


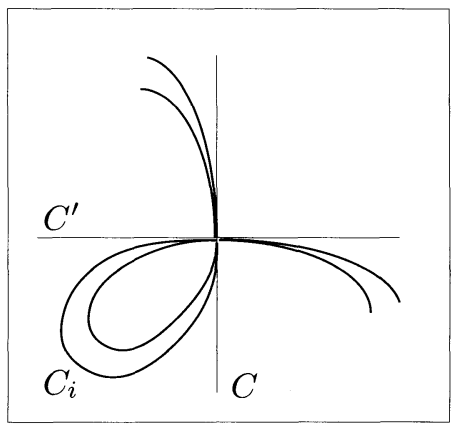

Figure 3.7

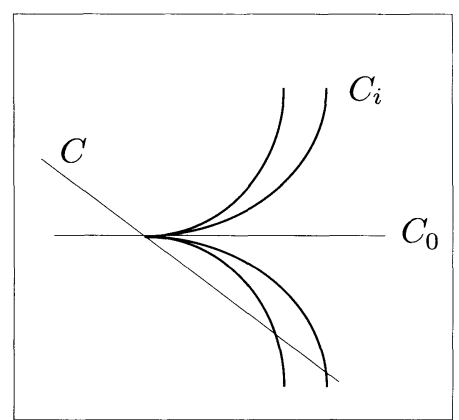

Figure 3.8

Proposition 3.4.

(i) Let $\operatorname{card} T=s+2$ where $s \geq 2$. Let $\bigcup_{i \in T} C_{i}$ be equivalent to $\left(\bigcup_{0 \leq i \leq s} C_{i}\right) \cup C$ with equations

$$
C_{i}: y^{k}=x^{k+1}+a_{i} y^{k+1} \quad \text { for } \quad i=1, \ldots, s
$$

where $k \geq 2$ and the $a_{i}$ are all different,

$$
C_{0}: y=0 \quad \text { and } \quad C: y=a x
$$

where $a \neq 0$ (Fig. 3.8). So $\chi(\stackrel{\circ}{E})=0$. Then $\mathcal{R}=0$.

(ii) Let $\operatorname{card} T=s+1$ where $s \geq 2$. Let $\bigcup_{i \in T} C_{i}$ be equivalent to $\bigcup_{0 \leq i \leq s} C_{i}$ as in (i). So $\chi(\stackrel{\circ}{E})=1-s$. Then $\mathcal{R}=0$.

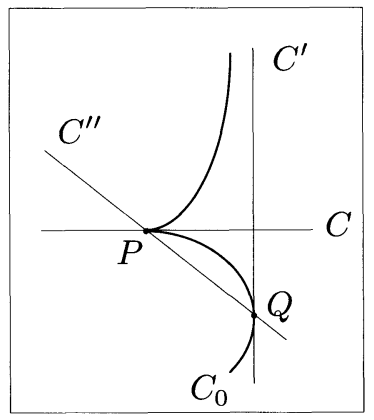

Figure 3.9

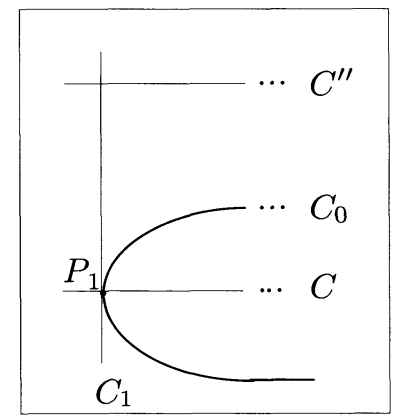

Figure 3.10

BULLETIN DE LA SOCIÉTÉ MATHÉMATIQUE DE FRANCE 


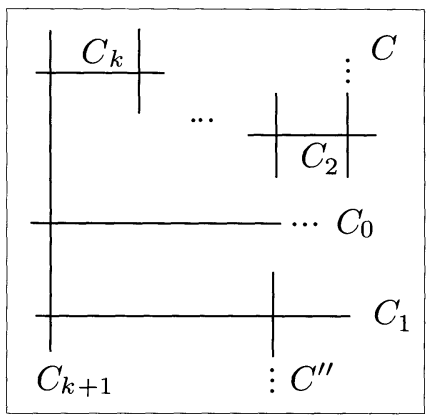

Figure 3.11

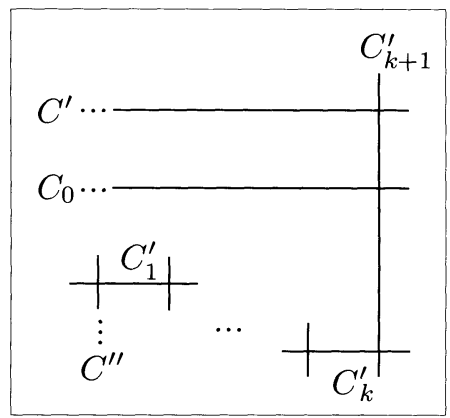

Figure 3.12

Proposition 3.5.

(i) Let $\operatorname{card} T=4$ and let $\bigcup_{i \in T} C_{i}$ be equivalent to $C_{0} \cup C \cup C^{\prime} \cup C^{\prime \prime}$ with equations

$$
\begin{aligned}
& C_{0}: y^{k} z=x^{k+1} \quad(\text { where } \quad k \geq 2), \\
& C: y=0, \quad C^{\prime}: z=0, \quad C^{\prime \prime}: x=0
\end{aligned}
$$

(figure 3.9 previous page). So $\chi(\stackrel{\circ}{E})=0$. Then $\mathcal{R}=0$.

(ii) Considering in (i) only curves equivalent to $C_{0} \cup C^{\prime}(\operatorname{card} T=2)$, $C_{0} \cup C \cup C^{\prime}$ or $C_{0} \cup C^{\prime} \cup C^{\prime \prime}(\operatorname{card} T=3)$ we also have $\chi(\stackrel{\circ}{E})=0$ and $\mathcal{R}=0$.

Proof.

(i) Let $C_{1}$ denote the exceptional curve of the blowing-up with center $P$ (Fig. 3.10 previous page). Since the intersection multiplicity of $C_{0}$ and $C_{1}$ at $P_{1}$ is $k$ we obtain an embedded resolution in a neighbourhood of $P$ by constructing next a series of $k$ blowing-ups (starting with center $P_{1}$ ) with exceptional curves $C_{2}, \ldots, C_{k+1}$ which intersect as given by figure 3.11 .

Analogously we have to construct in a neighbourhood of $Q$ a series of $k+1$ blowing-ups with exceptional curves $C_{1}^{\prime}, \ldots, C_{k+1}^{\prime}$ (Fig. 3.12). The intersection configuration of the $2 k+6$ curves forming a global embedded resolution of $\bigcup_{i \in T} C_{i}$ is presented in figure 3.13. Relation (B1) and relation (A) associated to $C_{1}$ yield respectively

$$
\begin{aligned}
& (k+1)\left(\alpha_{0}-1\right)+\alpha+\alpha^{\prime}+\alpha^{\prime \prime}=0, \\
& \alpha_{1}=k\left(\alpha_{0}-1\right)+\alpha+\alpha^{\prime \prime}
\end{aligned}
$$




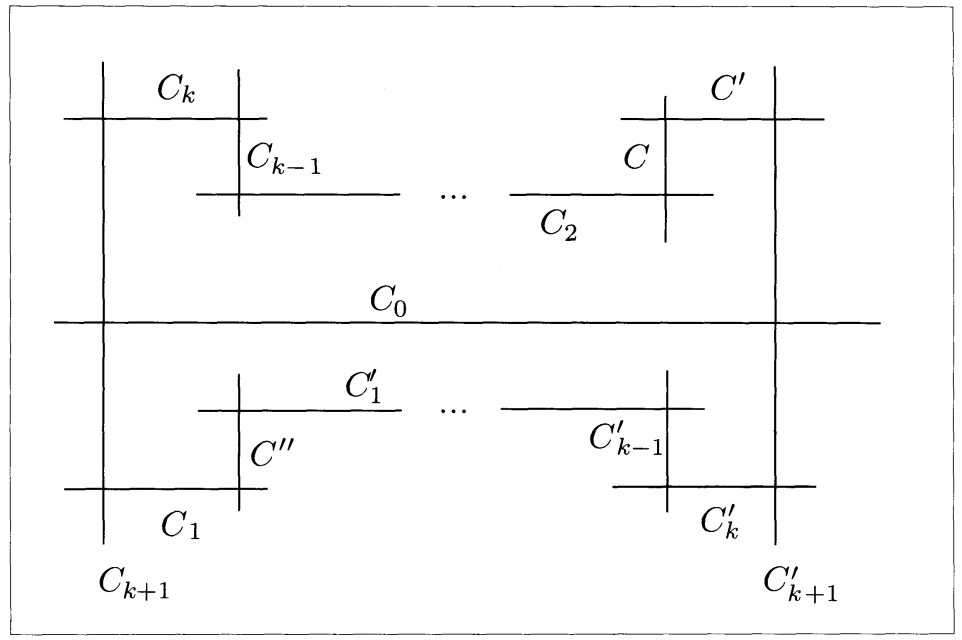

Figure 3.13

implying that

$$
\alpha_{1}+\alpha^{\prime}+\alpha_{0}-1=0
$$

By relation $(\mathrm{A})$ we have also :

(4) $\left\{\begin{array}{c}\alpha_{2}=\alpha_{0}+\alpha_{1}+\alpha-1, \\ \alpha_{3}=\alpha_{0}+\alpha_{1}+\alpha_{2}-1, \\ \alpha_{4}=\alpha_{0}+\alpha_{1}+\alpha_{3}-1, \\ \cdots \\ \alpha_{k}=\alpha_{0}+\alpha_{1}+\alpha_{k-1}-1, \\ \alpha_{k+1}=\alpha_{0}+\alpha_{1}+\alpha_{k}-1,\end{array} \quad\right.$ and $\quad\left\{\begin{array}{c}\alpha_{1}^{\prime}=\alpha_{0}+\alpha^{\prime}+\alpha^{\prime \prime}-1, \\ \alpha_{2}^{\prime}=\alpha_{0}+\alpha^{\prime}+\alpha_{1}^{\prime}-1, \\ \alpha_{3}^{\prime}=\alpha_{0}+\alpha^{\prime}+\alpha_{2}^{\prime}-1, \\ \cdots \\ \alpha_{k}^{\prime}=\alpha_{0}+\alpha^{\prime}+\alpha_{k-1}^{\prime}-1 \\ \alpha_{k+1}^{\prime}=\alpha_{0}+\alpha^{\prime}+\alpha_{k}-1\end{array}\right.$

Using (3) in the form $\alpha_{0}+\alpha_{1}-1=-\alpha^{\prime}$, respectively $\alpha_{0}+\alpha^{\prime}-1=-\alpha_{1}$, we can make (4) equivalent to :

(5)

$$
\left\{\begin{array} { c } 
{ \alpha = \alpha _ { 2 } + \alpha ^ { \prime } , } \\
{ \alpha _ { 2 } = \alpha _ { 3 } + \alpha ^ { \prime } , } \\
{ \alpha _ { 3 } = \alpha _ { 4 } + \alpha ^ { \prime } , } \\
{ \cdots } \\
{ \alpha _ { k - 1 } = \alpha _ { k } + \alpha ^ { \prime } , } \\
{ \alpha _ { k } = \alpha _ { k + 1 } + \alpha ^ { \prime } , }
\end{array} \quad \text { and } \quad \left\{\begin{array}{c}
\alpha^{\prime \prime}=\alpha_{1}^{\prime}+\alpha_{1}, \\
\alpha_{1}^{\prime}=\alpha_{2}^{\prime}+\alpha_{1}, \\
\alpha_{2}^{\prime}=\alpha_{3}^{\prime}+\alpha_{1}, \\
\cdots \\
\alpha_{k-1}^{\prime}=\alpha_{k}^{\prime}+\alpha_{1}, \\
\alpha_{k}^{\prime}=\alpha_{k+1}^{\prime}+\alpha_{1} .
\end{array}\right.\right.
$$




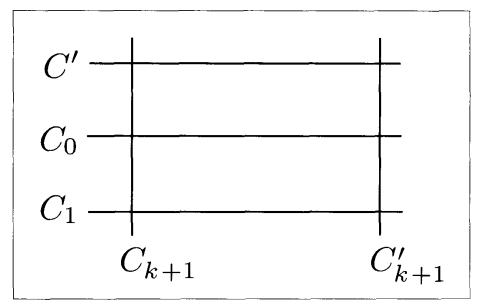

Figure 3.14

The $2 k+1$ relations of (5) and THEOREM 2.4 imply that we can blow down $2 k+1$ times to obtain the situation described by figure 3.14 . Now

$$
\begin{aligned}
\alpha_{k+1}+\alpha_{k+1}^{\prime} & =\left(\alpha-k \alpha^{\prime}\right)+\left(\alpha^{\prime \prime}-(k+1) \alpha_{1}\right) & & \text { by }(5) \\
& =\alpha+\alpha^{\prime \prime}-k \alpha^{\prime}+(k+1)\left(\alpha^{\prime}+\alpha_{0}-1\right) & & \text { by }(3) \\
& =(k+1)\left(\alpha_{0}-1\right)+\alpha+\alpha^{\prime}+\alpha^{\prime \prime} & & \\
& =0 & & \text { by }(1)
\end{aligned}
$$

and so $\mathcal{R}=0$ by Proposition 2.5.

(ii) These cases follow immediately from (i) by an application of Proposition 2.6.

\section{Singular points of multiplicity at most 4}

In this section we treat all possible intersection configurations on $E^{0}$ with $\chi(\stackrel{\circ}{E}) \leq 0$ when it is obtained by blowing-up a point of multiplicity $\mu=2,3$ or 4 on the strict transform of $Y$.

We first mention more generally (for arbitrary $n$ and $D$ ) that the intersections $C_{i}, i \in T$, on $E^{0}$ are «bounded》 by the multiplicity of $D$ on the strict transform of $Y$. Remember (Theorem 0.1) that $\Pi: E^{0} \rightarrow D$ is a projective space bundle over $D$ and that to every $C_{i}, i \in T$, we can associate a number $d_{i}$, which is the degree of the intersection cycle $C_{i} \cdot F$ on $F$ for a general fiber $F \cong \mathbb{P}^{n-\operatorname{dim} D}$ of $\Pi$.

Proposition 4.0.

(i) Let $Y^{(1)}$ and $Y^{(2)}$ respectively denote the strict transforms of $Y$ before and after the blowing-up (of the resolution $h$ ) with center $D$ and let $\mu$ be the multiplicity of any point of $D$ on $Y^{(1)}$. Then the numbers $d_{i}$, associated to the reduced irreducible components $C_{1}, \ldots, C_{s}$ of $Y^{(2)} \cap E^{0}$, 
satisfy

$$
d_{i} \geq 1 \quad \text { for } \quad i=1, \ldots, s \quad \text { and } \quad \sum_{i=1}^{s} d_{i} \leq \mu .
$$

(ii) Let $E_{\ell}^{(1)}$ and $E_{\ell}^{(2)}$ respectively denote the strict transforms of a previously created exceptional variety $E_{\ell}$ before and after the blowing-up with center $D$, and suppose that $D \subset E_{\ell}^{(1)}$. Then the intersection $E_{\ell}^{(2)} \cap E^{0}$ has associated number $d=1$.

We prove this proposition in the appendix and return now to the case $n=2$ and the data $\left.{ }^{*}\right)$ with $D$ a point and $E^{0} \cong \mathbb{P}^{2}$. In this case at most three previously created exceptional surfaces $E_{\ell}, \ell \in S^{\prime}$, contain $D$ (since $\bigcup_{\ell \in S^{\prime}} E_{\ell}$ has only normal crossings with $D$, see [Hi, p. 141]). Therefore by Proposition 4.0, using the same notations, the intersection of $E^{0}$ with $\left(\bigcup_{\ell \in S^{\prime}} E_{\ell}^{(2)}\right) \cup Y^{(2)}$ consists of at most three lines in general position (Fig. 2.2) and of a (possibly reducible) curve of degree at most $\mu$, where $\mu$ is the multiplicity of $D$ on $Y^{(1)}$.

When $\mu=2$ one can easily check that all possible configurations with $\chi(\stackrel{\circ}{E}) \leq 0$ consist of the six cases of Proposition 3.1 for $k=2,3$ or 4 and the four cases of Proposition 4.1 below.

Proposition 4.1.

(i) Let card $T=4$ and let $\bigcup_{i \in T} C_{i}$ be equivalent to $C \cup C_{0} \cup C_{1} \cup C_{1}^{\prime}$ with equations (Fig. 4.1 next page) :

$$
C: z^{2}=x y, \quad C_{0}: z=0, \quad C_{1}: x=0, \quad C_{1}^{\prime}: y=0 .
$$

So $\chi(\stackrel{\circ}{E})=0$. Then $\mathcal{R}=0$.

(ii) Considering in (i) only curves equivalent to $C \cup C_{1}(\operatorname{card} T=2)$, $C \cup C_{0} \cup C_{1}$ or $C \cup C_{1} \cup C_{1}^{\prime}(\operatorname{card} T=3)$ we also have $\chi(\stackrel{\circ}{E})=0$ and $\mathcal{R}=0$.

Proof.

(i) We obtain an embedded resolution of $C \cup C_{0} \cup C_{1} \cup C_{1}^{\prime}$ by constructing a series of two blowing-ups (starting with center $P$ ) with exceptional curves $C_{2}$ and $C_{3}$, and an analogous series (starting with center $Q$ ) with exceptional curves $C_{2}^{\prime}$ and $C_{3}^{\prime}$, which intersect as given by figure 4.2 next page. Relation (B1) and four times relation (A) yield respectively

$$
\begin{array}{ll}
2 \alpha+\alpha_{0}+\alpha_{1}+\alpha_{1}^{\prime}-2=0, & \\
\alpha_{2}=\alpha+\alpha_{0}+\alpha_{1}-1, & \alpha_{2}^{\prime}=\alpha+\alpha_{0}+\alpha_{1}^{\prime}-1, \\
\alpha_{3}=\alpha+\alpha_{1}+\alpha_{2}-1, & \alpha_{3}^{\prime}=\alpha+\alpha_{1}^{\prime}+\alpha_{2}^{\prime}-1,
\end{array}
$$




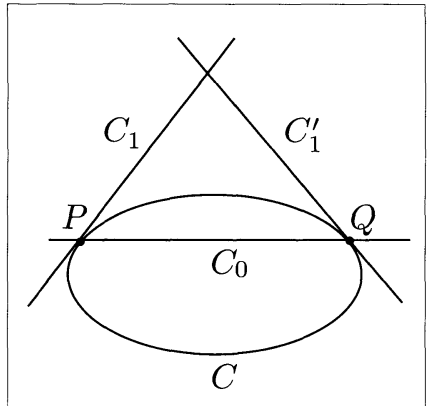

Figure 4.1

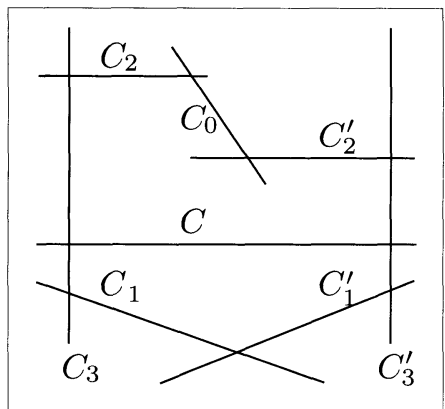

Figure 4.2

implying after a little calculation that :

$$
\begin{aligned}
& \alpha_{0}=\alpha_{2}+\alpha_{2}^{\prime}, \quad \alpha_{2}^{\prime}=\alpha_{2}+\alpha_{3}^{\prime}, \quad \alpha_{1}^{\prime}=\alpha_{1}+\alpha_{3}^{\prime}, \\
& \alpha_{3}+\alpha_{3}^{\prime}=0 .
\end{aligned}
$$

By Theorem 2.4 and the relations of (1) we can blow down three times to obtain the situation described by figure 4.3 . Then $\mathcal{R}=0$ by (2) and Proposition 2.5.

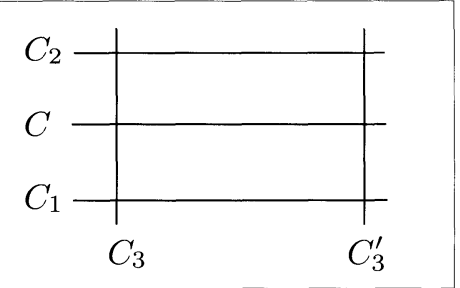

Figure 4.3

(ii) As usually these cases follow from (i) using Proposition 2.6.

When $\mu=3$ the possible configurations with $\chi(\stackrel{\circ}{E}) \leq 0$ consist of those occurring for $\mu=2$ and (other) configurations involving a curve $C$ of degree 3 and at most three lines in general position. One can easily verify that for an irreducible $C$ the only possible configurations are the three cases $(s=2, t=0),(s=1, t=0)$ and $(s=t=1)$ of Proposition 3.2 for $k=2$, and the four cases of Proposition 3.5 for $k=2$. For a reducible $C$ the (new) configurations consist of the two cases of Proposition 3.1 for $k=5$ and the case $k=2$ of Proposition 4.2 below. 
Proposition 4.2. - Let $\operatorname{card} T=k+2$ where $k \geq 2$, and let $\bigcup_{i \in T} C_{i}$ be equivalent to $C \cup C_{0} \cup\left(\bigcup_{1 \leq i \leq k} C_{i}\right)$ with equations

$$
C: z^{2}=x y, \quad C_{0}: y=0, \quad C_{i}: z=a_{i} y
$$

for $i=1, \ldots, k$ where the $a_{i}$ are all different (Fig. 4.4). So $\chi(\stackrel{\circ}{E})=0$. Then $\mathcal{R}=0$.

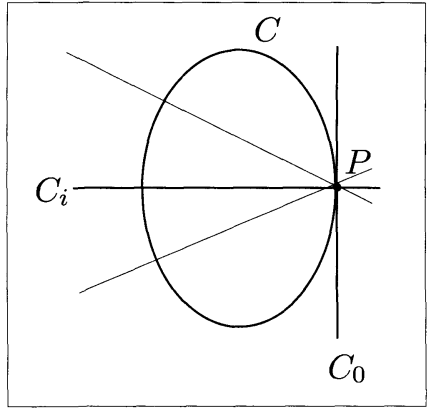

Figure 4.4

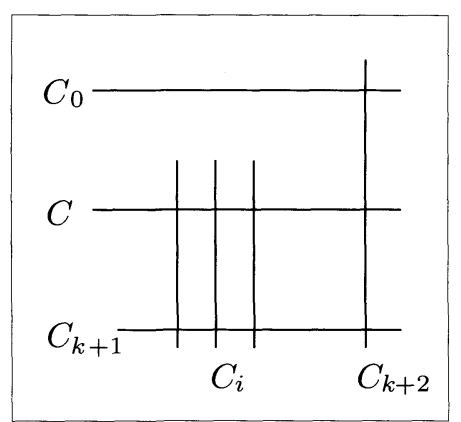

Figure 4.5

Proof. - We obtain an embedded resolution of $C \cup C_{0} \cup\left(\bigcup_{1 \leq i \leq k} C_{i}\right)$ by constructing two blowing-ups (starting with center $P$ ) with exceptional curves $C_{k+1}$ and $C_{k+2}$, which intersect as given by figure 4.5. Relation (B1) and two times relation (A) yield

$$
\left\{\begin{array}{l}
2(\alpha-1)+\left(\alpha_{0}-1\right)+\sum_{i=1}^{k}\left(\alpha_{i}-1\right)+3=0, \\
\alpha_{k+1}=(\alpha-1)+\left(\alpha_{0}-1\right)+\sum_{i=1}^{k}\left(\alpha_{i}-1\right)+2, \\
\alpha_{k+2}=\alpha+\alpha_{0}+\alpha_{k+1}-1,
\end{array}\right.
$$

implying that $\alpha_{k+1}+\alpha=0, \alpha_{0}=\alpha_{k+2}+1$. So blowing $C_{0}$ down (following Theorem 2.4) we see that $\mathcal{R}=0$ by Proposition 2.5.

When $\mu=4$ the possible configurations with $\chi(\stackrel{\circ}{E}) \leq 0$ consist of those occurring for $\mu=2$ or 3 and (other) configurations involving a curve $C$ of degree 4 and at most three lines in general position.

(a) If $C$ is irreducible and contains a point of multiplicity 3 , one can easily verify that the only possible configurations are the three cases $(s=2, t=0),(s=1, t=0)$ and $(s=t=1)$ of Proposition 3.2 for $k=3$, and the four cases of Proposition 3.5 for $k=3$. 
(b) If $C$ is irreducible and contains no point of multiplicity 3 , it can have at most three double points. When it has exactly three double points, one can easily see that a situation with $\chi(\stackrel{\circ}{E}) \leq 0$ could only occur if :

(1) $C$ has three ordinary cusps and an inflection point $P$ such that its tangent line (at $C$ ) intersects $C$ with intersection multiplicity 4 in $P$, or

(2) $C$ has two ordinary cusps and one (ordinary) node $P$ for which the two (principal) tangent lines at $C$ intersect $C$ with intersection multiplicity 4 in $P$.

Case (1) cannot occur by [EH, prop. 1.1]. Suppose that case (2) occurs. The bundle of lines through $P$ determines a base-point-free linear system of dimension 1 and degree 2 on $\mathbb{P}^{1}$, for which the associated morphism $\mathbb{P}^{1} \rightarrow \mathbb{P}^{1}$ of degree 2 has ramification degree at least 4 . This is impossible since this ramification degree must be 2 by Hurwitz's theorem.

When $C$ has exactly two or one double points one can verify that respectively the Propositions 4.3 and 4.4 describe all possible configurations with $\chi(\stackrel{\circ}{E}) \leq 0$. (We can prove both propositions by the usual techniques if we first «add》 the conic $y z+x^{2}=0$ to the configurations.)

\section{Proposition 4.3.}

(i) Let $\operatorname{card} T=3$ and let $\bigcup_{i \in T} C_{i}$ be equivalent to $C \cup C_{0} \cup C_{1}$ with equations (Fig. 4.6) :

$$
C:\left(y z+x^{2}\right)^{2}+x^{3} y=0, \quad C_{0}: y=0, \quad C_{1}: x=0 .
$$

So $\chi(\stackrel{\circ}{E})=0$. Then $\mathcal{R}=0$.

(ii) Let $\operatorname{card} T=2$ and let $\bigcup_{i \in T} C_{i}$ be equivalent to $C \cup C_{0}$ as in (i); then $\chi(\stackrel{\circ}{E})=0$ and $\mathcal{R}=0$.

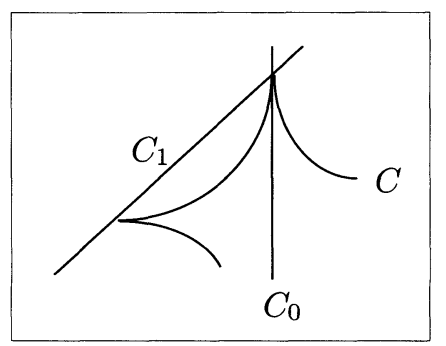

Figure 4.6

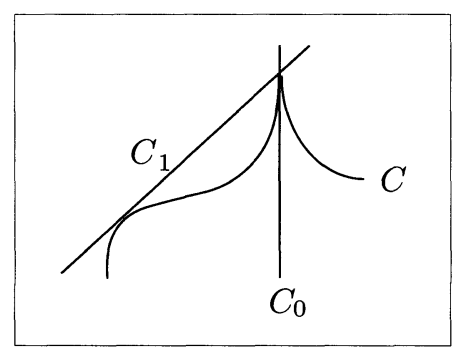

Figure 4.7

TOME $121-1993-\mathrm{N}^{\circ} 4$ 


\section{Proposition 4.4 .}

(i) Let $\operatorname{card} T=3$ and let $\bigcup_{i \in T} C_{i}$ be equivalent to $C \cup C_{0} \cup C_{1}$ with equations (Fig. 4.7) :

$$
C:\left(y z+x^{2}\right)^{2}+x y^{3}=0, \quad C_{0}: y=0, \quad C_{1}: x=0 .
$$

So $\chi(\stackrel{\circ}{E})=0$. Then $\mathcal{R}=0$.

(ii) Let $\operatorname{card} T=2$ and let $\bigcup_{i \in T} C_{i}$ be equivalent to $C \cup C_{0}$ as in (i); then $\chi(\stackrel{\circ}{E})=0$ and $\mathcal{R}=0$.

(c) Finally if $C$ is reducible one can verify that the only possible (new) configurations are the two cases of Proposition 3.1 for $k=6$, the two cases $(s=2, t=1)$ and $(s=1, t=2)$ of Proposition 3.2 for $k=2$, the case $k=3$ of Proposition 4.2, and the eight cases described in the Propositions 4.5, 4.7 and 4.8 below.

Proposition 4.5 .

(i) Let $\operatorname{card} T=4$ and let $\bigcup_{i \in T} C_{i}$ be equivalent to $C \cup C^{\prime} \cup C_{0} \cup C_{1}$ with equations (Fig. 4.8) :

$$
\begin{gathered}
C: y=x^{2}-y^{2}, \quad C_{0}: y=0, \\
C^{\prime}: y=x^{2}+y^{2}, \quad C_{1}: x=0 .
\end{gathered}
$$

So $\chi(\stackrel{\circ}{E})=0$. Then $\mathcal{R}=0$.

(ii) Let $\operatorname{card} T=3$ and let $\bigcup_{i \in T} C_{i}$ be equivalent to $C \cup C^{\prime} \cup C_{0}$ as in (i). Then $\chi(\stackrel{\circ}{E})=-1$ and $\mathcal{R}=0$.

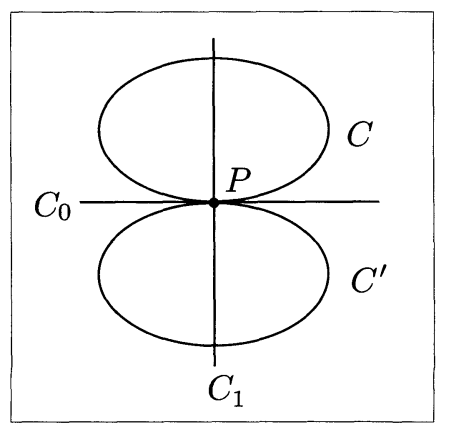

Figure 4.8

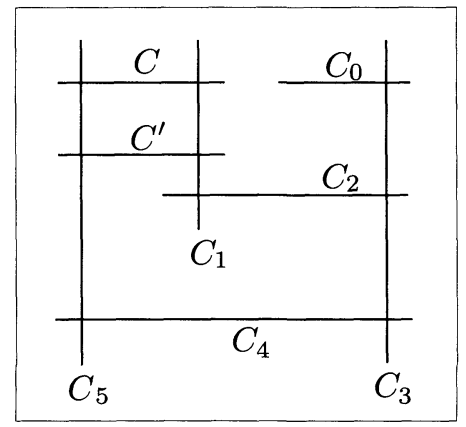

Figure 4.9 


\section{Proof.}

(i) We obtain an embedded resolution of $C \cup C^{\prime} \cup C_{0} \cup C_{1}$ by constructing a series of four blowing-ups (starting with center $P$ ) with exceptional curves $C_{i}, 2 \leq i \leq 5$, which intersect as given by figure 4.9 on previous page. Relation (B1) and four times relation (A) yield respectively :

$$
\left\{\begin{array} { l } 
{ 2 \alpha + 2 \alpha ^ { \prime } + \alpha _ { 0 } + \alpha _ { 1 } - 3 = 0 , } \\
{ \alpha _ { 2 } = \alpha + \alpha ^ { \prime } + \alpha _ { 0 } + \alpha _ { 1 } - 2 , } \\
{ \alpha _ { 3 } = \alpha + \alpha ^ { \prime } + \alpha _ { 0 } + \alpha _ { 2 } - 2 , } \\
{ \alpha _ { 4 } = \alpha + \alpha ^ { \prime } + \alpha _ { 3 } - 1 , } \\
{ \alpha _ { 5 } = \alpha + \alpha ^ { \prime } + \alpha _ { 4 } - 1 , }
\end{array} \quad \text { implying that } \quad \left\{\begin{array}{l}
\alpha_{0}=\alpha_{3}+1 \\
\alpha_{3}=\alpha_{2}+\alpha_{4} \\
\alpha_{4}=\alpha_{2}+\alpha_{5} \\
\alpha_{1}+\alpha_{5}=0
\end{array}\right.\right.
$$

So by TheORem 2.4 we can blow down three times to obtain the situation described by figure 4.10 and then $\mathcal{R}=0$ by Proposition 2.5 .

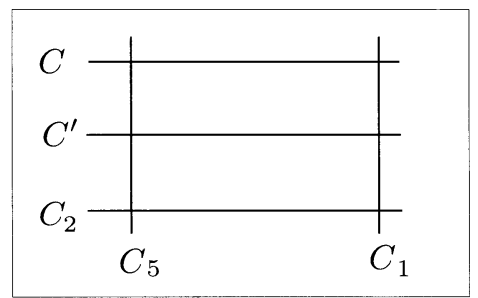

Figure 4.10

(ii) As usually this case follows from (i) by Proposition 2.6.

Remark 4.6. - In Proposition 4.5 the configuration $C \cup C^{\prime}$ determines another case where $\chi(\stackrel{\circ}{E})=0$, but it is not relevant because then necessarily $\alpha_{3}=0$ (using the same notations as in the proof above).

We can analogously prove the next two propositions.

Proposition 4.7.

(i) Let card $T=5$ and let $\bigcup_{i \in T} C_{i}$ be equivalent to $C \cup C^{\prime} \cup C_{0} \cup C_{1} \cup C_{2}$ with equations (Fig. 4.11):

$$
\begin{array}{ll}
C: y z=x^{2}, & C^{\prime}: y z=-x^{2}, \\
C_{0}: x=0, & C_{1}: y=0, \quad C_{2}: z=0 .
\end{array}
$$

So $\chi(\stackrel{\circ}{E})=0$. Then $\mathcal{R}=0$.

TOME $121-1993-\mathrm{N}^{\circ} 4$ 
(ii) Considering in (i) instead of $C_{0} \cup C_{1} \cup C_{2}$ only $C_{1}$ ( $\operatorname{card} T=3$ ), $C_{0} \cup C_{1}$ or $C_{1} \cup C_{2}(\operatorname{card} T=4)$, we also have $\chi(\stackrel{\circ}{E})=0$ and $\mathcal{R}=0$.

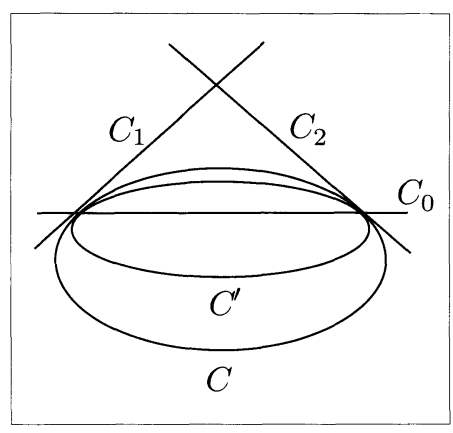

Figure 4.11

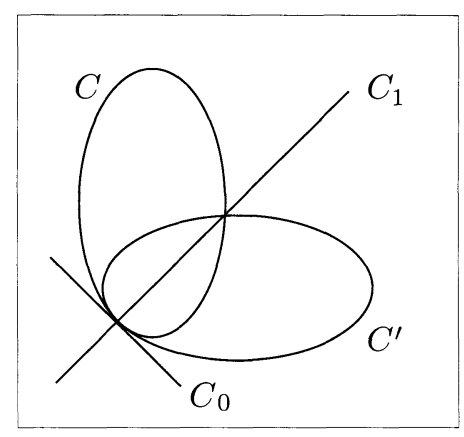

Figure 4.12

Proposition 4.8 .

(i) Let card $T=4$ and let $\bigcup_{i \in T} C_{i}$ be equivalent to $C \cup C^{\prime} \cup C_{0} \cup C_{1}$ with equations (Fig. 4.12):

$$
\begin{aligned}
C: y z=x^{2}+x y, & C_{0}: y=0 \\
C^{\prime}: y z=x^{2}-x y, & C_{1}: x=0 .
\end{aligned}
$$

So $\chi(\stackrel{\circ}{E})=0$. Then $\mathcal{R}=0$.

(ii) Let $\operatorname{card} T=3$ and let $\cup_{i \in T} C_{i}$ be equivalent to $C \cup C^{\prime} \cup C_{0}$ as in $(\mathrm{i})$; then $\chi(\stackrel{\circ}{E})=0$ and $\mathcal{R}=0$.

We can summarize the previous propositions of this section in the following theorem.

TheORem 4.9. - If $E_{j}$ in the data (*) of paragraph 1 is created by blowing-up a point of multiplicity at most 4 on the strict transform of $Y$, then $\mathcal{R}=0$.

Corollary 4.10. - If the surface $Y$ in $\mathbb{A}^{3}$ has only singular points of multiplicity at most 4 , then $\mathcal{R}=0$ for all $E_{j}$ in the data $\left(^{*}\right)$ created by blowing-up a point.

Now to conclude this section we state some interesting examples of configurations with $\chi(\stackrel{\circ}{E}) \leq 0$ involving a curve $C$ of degree 5 and at most 
three lines in general position. (We can prove that $\mathcal{R}=0$ in these cases by the usual techniques.) When $C$ is reducible with an (irreducible) conic and cubic as components one can show by tedious calculations on the equations of those curves in $\mathbb{P}^{2}$ that the only possible configurations are described in the Propositions 4.11 and 4.12 below.

Proposition 4.11.

(i) Let $\operatorname{card} T=4$ and let $\bigcup_{i \in T} C_{i}$ be equivalent to $\bigcup_{1 \leq i \leq 4} C_{i}$ with equations where $a \neq 0$ (Fig. 4.13) :

$$
\begin{array}{ll}
C_{1}: y^{2}=x^{3}, & C_{3}: y=0, \\
C_{2}: y=3 a x^{2}-3 a^{2} x y+a^{3} y^{2}, & C_{4}: x=a y
\end{array}
$$

So $\chi(\stackrel{\circ}{E})=0$. Then $\mathcal{R}=0$.

(ii) Let $\operatorname{card} T=3$ and let $\bigcup_{i \in T} C_{i}$ be equivalent to $\bigcup_{1 \leq i \leq 3} C_{i}$ as in (i). Then $\chi(\stackrel{\circ}{E})=0$ and $\mathcal{R}=0$.

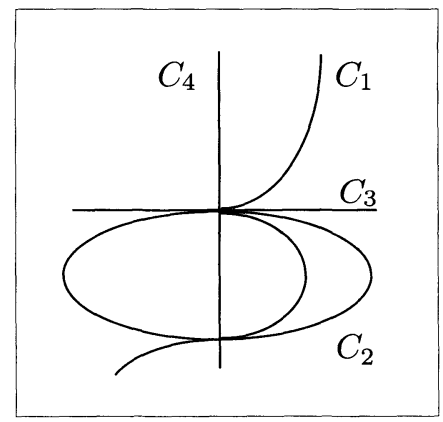

Figure 4.13

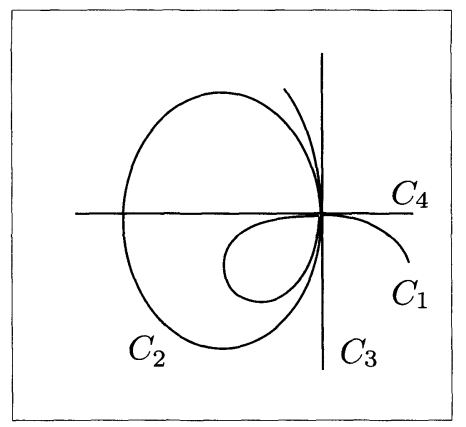

Figure 4.14

Proposition 4.12.

(i) Let $\operatorname{card} T=4$ and let $\bigcup_{i \in T} C_{i}$ be equivalent to $\bigcup_{i \leq i \leq 4} C_{i}$ with equations (Fig. 4.14):

$$
\begin{array}{ll}
C_{1}: y^{2}-x^{2}=y^{3}, & C_{3}: y=x, \\
C_{2}: 8(y-x)=x^{2}-4 x y+7 y^{2}, & C_{4}: y=-x
\end{array}
$$

So $\chi(\stackrel{\circ}{E})=0$. Then $\mathcal{R}=0$.

(ii) Let card $T=3$ and let $\bigcup_{i \in T} C_{i}$ be equivalent to $\bigcup_{1 \leq i \leq 3} C_{i}$ as in (i). Then $\chi(\stackrel{\circ}{E})=0$ and $\mathcal{R}=0$. 
The most simple example of a configuration with $\chi(\stackrel{\circ}{E}) \leq 0$ involving a curve with two different singular points is the following. It is remarkable that the (local) resolution data of the two singular points must interact in order to have $\mathcal{R}=0$.

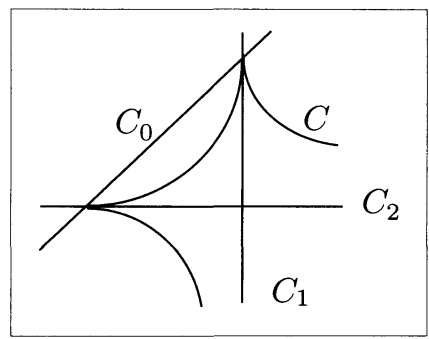

Figure 4.15

Proposition 4.13.

(i) Let $\operatorname{card} T=4$ and let $\bigcup_{i \in T} C_{i}$ be equivalent to $C \cup C_{0} \cup C_{1} \cup C_{2}$ with equations (Fig. 4.15) :

$$
\begin{array}{ll}
C: y^{2} z^{3}=x^{5}, & C_{1}: y=0 \\
C_{0}: x=0, & C_{2}: z=0 .
\end{array}
$$

So $\chi(\stackrel{\circ}{E})=0$. Then $\mathcal{R}=0$.

(ii) Considering in (i) only $C \cup C_{1}, C \cup C_{2}(\operatorname{card} T=2), C \cup C_{0} \cup C_{1}$, $C \cup C_{0} \cup C_{2}$ or $C \cup C_{1} \cup C_{2}(\operatorname{card} T=3)$, we also have $\chi(\stackrel{\circ}{E})=0$ and $\mathcal{R}=0$.

Remark 4.14. - Proposition 4.13 can be generalized to an arbitrary number of curves $C_{i}: y^{2} z^{3}=a_{i} x^{5}$ (with different $a_{i}$ ).

REMARK 4.15. - In [Si, example 7.6] also SiERsma classifies projective plane curves $C$ satisfying $\chi\left(\mathbb{P}^{2} \backslash C\right)=0$. He lists the examples for which $\operatorname{deg} C \leq 4$, producing thus some cases of the list in this section.

His motivation is the following. Let the homogeneous polynomial $g(x, y, z)$ determine the germ of an analytic function $g:\left(\mathbb{C}^{3}, 0\right) \rightarrow(\mathbb{C}, 0)$ with 1-dimensional singular locus. A necessary condition for the Milnor monodromy $T_{2}$ in the origin being the identity is that $\chi\left(\mathbb{P}^{2} \backslash\{g=0\}\right)=0$. 


\section{Starting with a ruled surface}

In this section we suppose that $D$ is a nonsingular curve and thus that $E^{0}$ is a projective space bundle $\Pi: E^{0} \rightarrow D$ or ruled surface over $D$. To every irreducible curve $C$ on $E^{0}$ we can associate a natural number $d$ which is the number of intersection points of $C$ with a general fiber of $\Pi$; we will talk in this context of the associated number $d$ of $C$. In particular $d=0$ if and only if $C$ itself is a fiber of $\Pi$.

REMARK 5.0. - It is a fact that not every configuration of curves $C_{i}$, $i \in T$, can occur on $E^{0}$ (see [V2, example 7.3] and Proposition 5.8 and compare with remark 3.0 (iii)). For «many $》$ cases treated in this section however there exist concrete examples of surfaces $Y$ in $\mathbb{A}^{3}$ for which these cases occur in the resolution process.

We first mention two basic examples, which can be considered as generalizations of PROPOSITION 1.5.

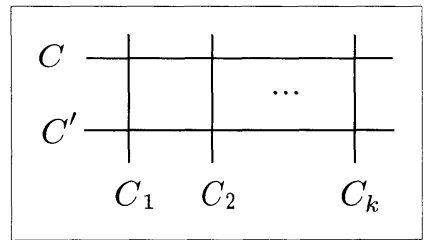

Figure 5.1

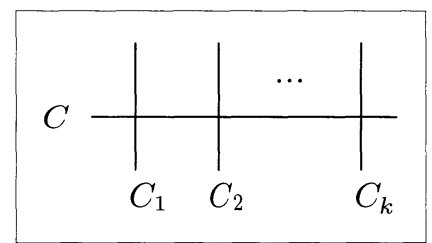

Figure 5.2

Proposition 5.1. - Let $k \geq 0$ and let $C_{i}, i \leq 1 \leq k$, be fibers of $\Pi$.

(i) Let $\operatorname{card} T=k+2$, let $C$ and $C^{\prime}$ have associated number $d=d^{\prime}=1$ and let $C \cap C^{\prime}=\emptyset$ (Fig. 5.1). So $\chi(\stackrel{\circ}{E})=0$. Or

(ii) let $\operatorname{card} T=k+1$ and let $C$ have associated number $d=1$ (Fig. 5.2). So $\chi(\stackrel{\circ}{E})=\chi(D)-k$.

Then $\mathcal{R}=0$.

Proof. - Clearly $\bigcup_{i \in T} C_{i}$ has normal crossings on $E^{0}$ and these cases are examples of Proposition 2.5 since by example 0.4 (2) (of relation (B1)) we have $\alpha+\alpha^{\prime}=0$ in case (i) and $\alpha=-1$ in case (ii).

We now study the configurations occurring when the strict transform of $Y$ before the blowing-up with center $D$ is already nonsingular.

We use for a while the notations of Proposition 4.0. We have that exactly zero, one or two previously created exceptional surfaces $E_{i}^{(1)}$,

TOME $121-1993-\mathrm{N}^{\circ} 4$ 
$i \in S^{\prime}$, contain $D$ (since $\bigcup_{i \in S^{\prime}} E_{i}^{(1)}$ has normal crossings with $D$, see [Hi, p. 141]). Also in the last case the two curves $E^{0} \cap E_{i}^{(2)}$ on $E^{0}$ are disjoint. Moreover by Proposition 4.0 the numbers $d$ associated to those curves $E^{0} \cap E_{i}^{(2)}$ and to $E^{0} \cap Y^{(2)}$ are equal to 1 . We thus have (after some thinking) :

Proposition 5.2. - When $D$ is a curve and the strict transform of $Y$ before the blowing-up with center $D$ is already nonsingular, then for the $C_{i}, i \in T$, only the intersection configurations described by the figures 5.2 (case 1), 5.3 (case 2) and 5.4 (case 3) can eventually occur on $E^{0}$. Here $k \geq 0$, the curves $C_{i}, 1 \leq i \leq k$, are fibers and the curves $C, C^{\prime}$ and $C^{\prime \prime}$ have associated numbers $d=d^{\prime}=d^{\prime \prime}=1$.

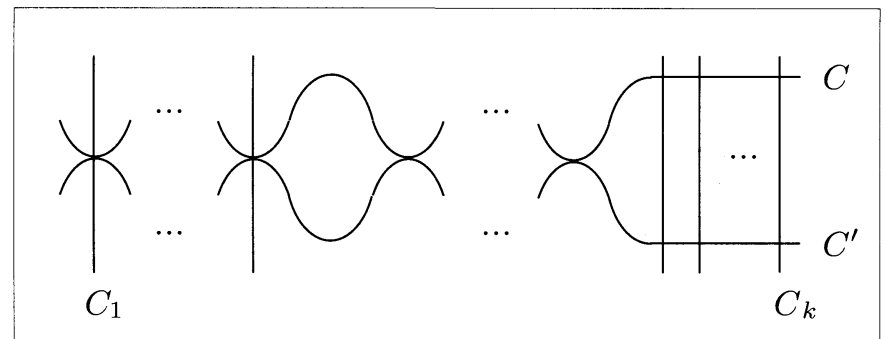

Figure 5.3 (case 2)

Here the curves $C$ and $C^{\prime}$ do not have to be tangent in their intersection points and can eventually be disjoint.

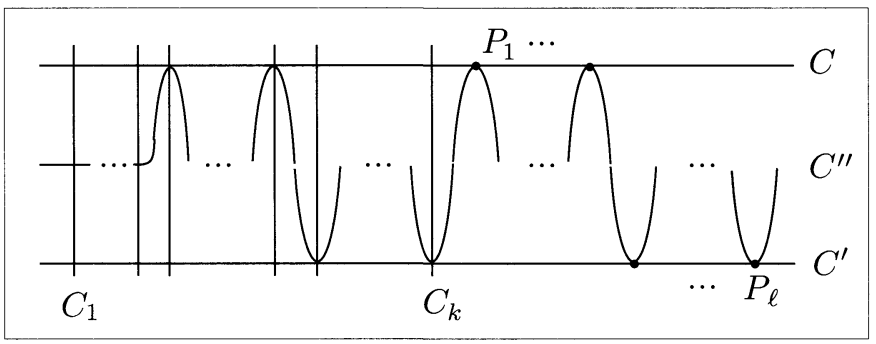

Figure 5.4 (case 3)

Here the curves $C$ and $C^{\prime \prime}$, resp. $C^{\prime}$ and $C^{\prime \prime}$, do not have to be tangent in their intersection points and can eventually be disjoint.

For case 1 of Proposition 5.2 we have that $\mathcal{R}=0$ in the data $\left(^{*}\right)$ by Proposition 5.1 (ii). For case 2 we easily see that $\chi(\stackrel{\circ}{E}) \leq 0$ 
$\left(\Leftrightarrow \chi(\stackrel{\circ}{E})=0\right.$ ) if and only if each point of $C \cap C^{\prime}$ is contained in some fiber $C_{i}$ for $i=1, \ldots, k$. Now if $C \cap C^{\prime}=\emptyset$ we have that $\mathcal{R}=0$ by Proposition 5.1 (i). If $C \cap C^{\prime} \neq \emptyset$ then $\mathcal{R}=0$ by Proposition 5.3 below.

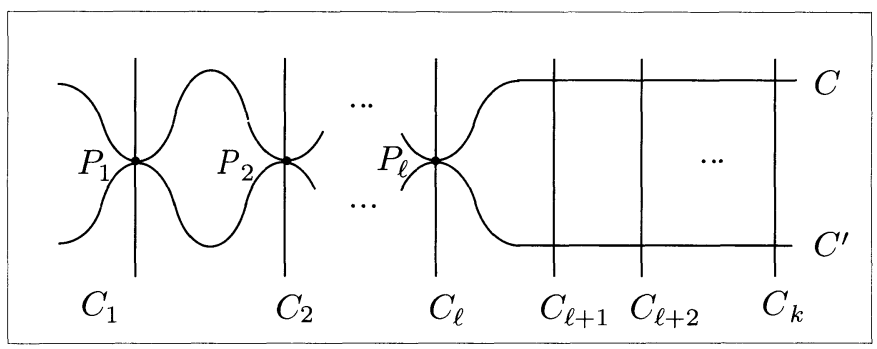

Figure 5.5

Proposition 5.3. - Let $\operatorname{card} T=k+2$ where $k \geq 1$. Let $C_{i}, 1 \leq i \leq k$, be fibers and let $C$ and $C^{\prime}$ have associated numbers $d=d^{\prime}=1$. Suppose also that $\operatorname{card}\left(C \cap C^{\prime}\right)=\ell$ where $\ell \geq 1$, and that each point $P_{i}, 1 \leq i \leq \ell$, of $C \cap C^{\prime}$ belongs to the fiber $C_{i}$ (Fig. 5.5). So $\chi(\stackrel{\circ}{E})=0$. Then $\mathcal{R}=0$.

Proof. - For $i=1, \ldots, \ell$ let $n_{i}$ be the intersection multiplicity of $C$ and $C^{\prime}$ at $P_{i}$. We obtain an embedded resolution of $C \cup C^{\prime} \cup\left(\cup_{1 \leq i \leq k} C_{i}\right)$ by constructing for $i=1, \ldots, \ell$ a series of $n_{i}$ blowing-ups (starting with center $P_{i}$ ). By the same argument as in the proof of Proposition 3.2 and using that $\alpha+\alpha^{\prime}=0$ (by relation (B1)) we have that $\mathcal{R}=0$.

We now determine when $\chi(\stackrel{\circ}{E}) \leq 0$ in case 3 of Proposition 5.2 if $D$ is projective. Let $C^{\prime \prime}$ intersect $C \cup C^{\prime}$ in $\ell$ different points outside $\bigcup_{1 \leq i \leq k} C_{i}$ (Fig. 5.4). Then

$$
\begin{aligned}
\chi(\stackrel{\circ}{E}) & =2(2-2 g)-[2(2-2 g)+(2-2 g-k-\ell)+k(2-2)] \\
& =2 g-2+k+\ell,
\end{aligned}
$$

where $g$ is the genus of $D$, and so $\chi(\stackrel{\circ}{E}) \leq 0$ if and only if either

$$
\left\{\begin{array} { l } 
{ g = 0 , } \\
{ k + \ell \leq 2 , }
\end{array} \text { or } \quad \left\{\begin{array}{l}
g=1, \\
k=\ell=0 .
\end{array}\right.\right.
$$

In the Propositions 5.4 and 5.6 below we prove that $\mathcal{R}=0$ in case 3 of Proposition 5.2 for a projective $D$ except for two special cases.

TOME $121-1993-\mathrm{N}^{\circ} 4$ 


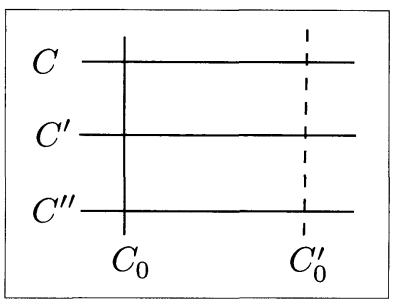

Figure 5.6

Proposition 5.4. - Suppose that $D \cong \mathbb{P}^{1}$. Let $C, C^{\prime}$ and $C^{\prime \prime}$ be nonintersecting curves with associated numbers $d=d^{\prime}=d^{\prime \prime}=1$.

(i) Let $\operatorname{card} T=5$ and let $C_{0}$ and $C_{0}^{\prime}$ be fibers of $\Pi$ (Fig. 5.6). So $\chi(\stackrel{\circ}{E})=0$. Or

(ii) let $\operatorname{card} T=4$ and let $C_{0}$ be a fiber of $\Pi$ (Fig. 5.6). So $\chi(\stackrel{\circ}{E})=-1$. Then $\mathcal{R}=0$.

Proof.

(i) Since this is an example of Proposition 2.5 we only have to prove that $\alpha_{0}+\alpha_{0}^{\prime}=0$. By example 0.5 the self-intersection numbers of $C, C^{\prime}$ and $C^{\prime \prime}$ are zero and so example 0.4 (2) (of relation (B2)) then yields $\left(\alpha_{0}-1\right)+\left(\alpha_{0}^{\prime}-1\right)=-2$.

(ii) This case can be proved analogously or by an application of Proposition 2.6.

REMARK 5.5.- In the situation above we cannot derive the case without fibers ( $\operatorname{card} T=3$ ) by setting $\alpha_{0}=1$ in the $\mathcal{R}$ of case (ii) since $\alpha_{0}=-1$ for the configuration of case (ii). (See also Proposition 5.8.)

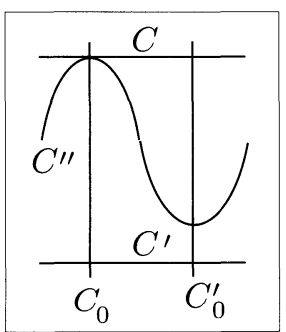

Figure 5.7

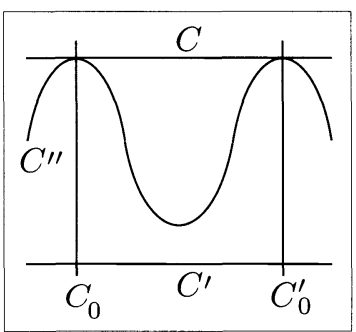

Figure 5.8

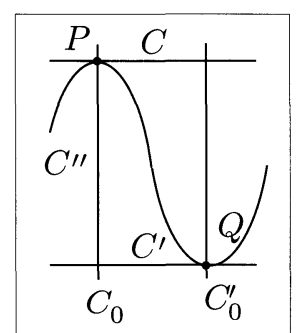

Figure 5.9

Here the curves $C$ and $C^{\prime \prime}$, resp. $C^{\prime}$ and $C^{\prime \prime}$, do not have to be tangent in their intersection points. 
Proposition 5.6. - Suppose that $D \cong \mathbb{P}^{1}$. Let the curves $C, C^{\prime}$ and $C^{\prime \prime}$ have associated numbers $d=d^{\prime}=d^{\prime \prime}=1$ and let them intersect as given by the figures $5.7,5.8$ or 5.9 on previous page. Let $\operatorname{card} T=3,4$ or 5 according to zero, one or two of the fibers $C_{0}$ or $C_{0}^{\prime}$ occur on $E^{0}$. (These conditions yield ten different configurations.) Then $\mathcal{R}=0$, except when $\operatorname{card} T=3$ and $C \cup C^{\prime} \cup C^{\prime \prime}$ has normal crossings in figure 5.8 (see figure 5.12).

Proof. - The situations with card $T=3$ or 4 follow easily by Proposition 2.6 from the situation with $\operatorname{card} T=5$. We will only prove that $\mathcal{R}=0$ for the situation of figure 5.9, the other two proofs being similar.

Let $n$ and $m$ be the intersection multiplicities of respectively $C$ and $C^{\prime \prime}$ at $P$, and $C^{\prime}$ and $C^{\prime \prime}$ at $Q$. Then we obtain an embedded resolution of $C \cup C^{\prime} \cup C^{\prime \prime} \cup C_{0} \cup C_{0}^{\prime}$ by constructing a series of $n$ blowing-ups with exceptional curves $C_{1}, \ldots, C_{n}$ (starting with center $P$ ) and another series of $m$ blowing-ups with exceptional curves $C_{1}^{\prime}, \ldots, C_{m}^{\prime}$ (starting with center $Q$ ), which intersect as given by figure 5.10 .

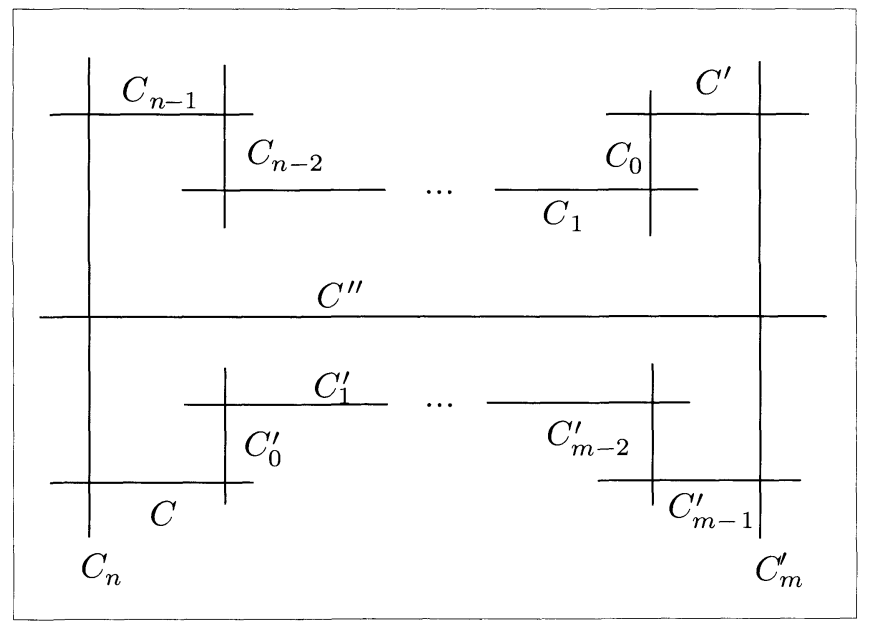

Figure 5.10

By example 0.5 the self-intersection numbers of $C, C^{\prime}$ and $C^{\prime \prime}$ are respectively $n-m, m-n$ and $m+n$. Therefore the relations (B1) and (B2) yield respectively

$$
\alpha+\alpha^{\prime}+\alpha^{\prime \prime}-1=0,
$$

$$
\text { and } \begin{aligned}
\frac{1}{2}(n-m)(\alpha-1)+\frac{1}{2}(m-n)\left(\alpha^{\prime}-1\right) & +\frac{1}{2}(m+n)\left(\alpha^{\prime \prime}-1\right) \\
& +\left(\alpha_{0}-1\right)+\left(\alpha_{0}^{\prime}-1\right)=-2,
\end{aligned}
$$

TOME $121-1993-\mathrm{N}^{\circ} 4$ 
which we can write more elegantly (using (1)) as

$$
m \alpha+n \alpha^{\prime}=\alpha_{0}+\alpha_{0}^{\prime}
$$

The $n+m$ relations (A) yield

$$
\left\{\begin{array} { l } 
{ \alpha _ { 1 } = \alpha + \alpha ^ { \prime \prime } + \alpha _ { 0 } - 1 , } \\
{ \alpha _ { 2 } = \alpha + \alpha ^ { \prime \prime } + \alpha _ { 1 } - 1 , } \\
{ \ldots } \\
{ \alpha _ { n - 1 } = \alpha + \alpha ^ { \prime \prime } + \alpha _ { n - 2 } - 1 , } \\
{ \alpha _ { n } = \alpha + \alpha ^ { \prime \prime } + \alpha _ { n - 1 } - 1 , }
\end{array} \quad \text { and } \quad \left\{\begin{array}{c}
\alpha_{1}^{\prime}=\alpha^{\prime}+\alpha^{\prime \prime}+\alpha_{0}^{\prime}-1, \\
\alpha_{2}^{\prime}=\alpha^{\prime}+\alpha^{\prime \prime}+\alpha_{1}^{\prime}-1, \\
\ldots \\
\alpha_{m-1}^{\prime}=\alpha^{\prime}+\alpha^{\prime \prime}+\alpha_{m-2}^{\prime}-1, \\
\alpha_{m}^{\prime}=\alpha^{\prime}+\alpha^{\prime \prime}+\alpha_{m-1}^{\prime}-1,
\end{array}\right.\right.
$$

and thus, using (1), also

(3)

$$
\left\{\begin{array} { l } 
{ \alpha _ { 0 } = \alpha _ { 1 } + \alpha ^ { \prime } , } \\
{ \alpha _ { 1 } = \alpha _ { 2 } + \alpha ^ { \prime } , } \\
{ \ldots } \\
{ \alpha _ { n - 2 } = \alpha _ { n - 1 } + \alpha ^ { \prime } , } \\
{ \alpha _ { n - 1 } = \alpha _ { n } + \alpha ^ { \prime } }
\end{array} \quad \text { and } \quad \left\{\begin{array}{c}
\alpha_{0}^{\prime}=\alpha_{1}^{\prime}+\alpha \\
\alpha_{1}^{\prime}=\alpha_{2}^{\prime}+\alpha \\
\ldots \\
\alpha_{m-2}^{\prime}=\alpha_{m-1}^{\prime}+\alpha \\
\alpha_{m-1}^{\prime}=\alpha_{m}^{\prime}+\alpha
\end{array}\right.\right.
$$

By (3) and Theorem 2.4 we can blow down $n+m$ times to obtain the situation described by figure 5.11. Using (3) and (2) we can compute that

$$
\alpha_{n}+\alpha_{m}^{\prime}=\left(\alpha_{0}-n \alpha^{\prime}\right)+\left(\alpha_{0}^{\prime}-m \alpha\right)=0
$$

and so by Proposition 2.5 we obtain that $\mathcal{R}=0$.

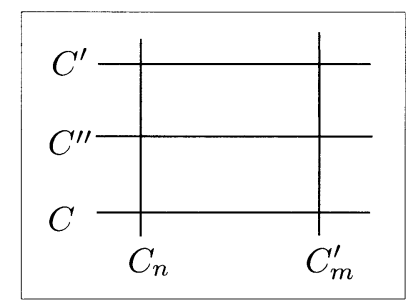

Figure 5.11

\section{REMARK 5.7.}

(a) For the exceptional configuration (Fig. 5.12 next page) of PROPOSITION 5.6 we cannot derive that $\mathcal{R}=0$ from the fact that $\mathcal{R}=0$ for the analogous configuration (Fig. 5.13 next page) with one fiber $C_{0}$ added! The method of Proposition 2.6 fails in this case because substituting $\alpha_{0}=1$ in the data of the configuration with $C_{0}$ yields $\alpha_{1}=0$, where $C_{1}$ is the exceptional curve of the blowing-up with center $P$. 


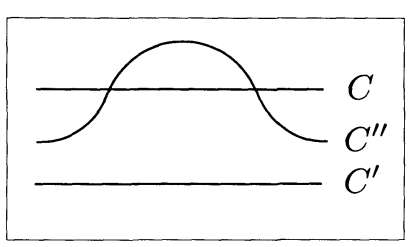

Figure 5.12 (exceptional case)

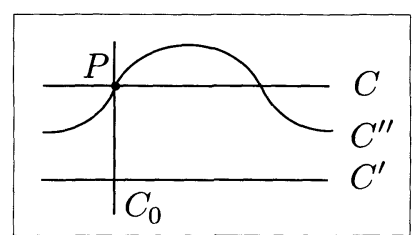

Figure 5.13

(b) We have in fact that $\mathcal{R} \neq 0$ for this exceptional configuration. Now the relations (B1) and (B2) imply that $\alpha^{\prime}=1$ and thus that $\left(\nu^{\prime}-1\right) / N^{\prime}=\nu_{j} / N_{j}$ where $\left(N^{\prime}, \nu^{\prime}\right)$ are the numerical data of the exceptional surface that intersects $E_{j}$ in (the strict transform of) $C^{\prime}$. So $N_{j}^{\prime} \mid N^{\prime}$, where $\nu_{j} / N_{j}=\nu_{j}^{\prime} / N_{j}^{\prime}$ and $\nu_{j}^{\prime}$ and $N_{j}^{\prime}$ are coprime, and in view of remark 1.4 (i) there is no obstruction for the monodromy conjecture.

(c) For $f(x, y, z)=x^{2}-y^{2}+z^{3}$ this exceptional situation actually occurs.

Until now we have considered in case 3 of Proposition 5.2 for a projective $D$ all configurations with $\chi(\stackrel{\circ}{E}) \leq 0$, except the configuration with $k=\ell=0$ (eventually occurring when the genus of $D$ is 0 or 1 ). By the following proposition this configuration is not possible if the genus of $D$ is 0 .

Proposition 5.8. - When $D$ is projective the configuration with $k=\ell=0$ cannot occur in case 3 of Proposition 5.2 if $g \neq 1$, where $g$ is the genus of $D$.

Proof. - By example 0.5 the self-intersection numbers of $C, C^{\prime}$ and $C^{\prime \prime}$ are zero; example $0.4(2)$ then yields that $0=2 g-2$, which is impossible for $g \neq 1$.

Summing up, the Propositions 5.2 to 5.8 imply :

THEOREM 5.9. - Suppose that $E_{j}$ in the data $\left(^{*}\right)$ of paragraph 1 is created by blowing-up a projective curve $D$ and that the strict transform of $Y$ before this blowing-up is nonsingular. Except for

(1) the situation described by figure 5.12 and

(2) the situation when the genus of $D$ is 1 and the $C_{i}, i \in T$, consist of three nonintersecting curves with associated number $d_{i}=1$,

we have that $\mathcal{R}=0$. 
For absolutely isolated singularities we have a sharper result.

Definition 5.10. - A singular point $P$ of a reduced surface $V$ (embedded in some nonsingular threefold) is absolutely isolated if there exists a resolution of $V$ in a neighbourhood of $P$ obtained only by blowingups with a point as center.

We now suppose that if all singular points of the surface $Y$ are absolutely isolated then, until the strict transform of $Y$ is nonsingular, the resolution process consists only of blowing-ups with a point as center.

COROLlary 5.11. - Let all singular points of the surface $Y$ in $\mathbb{A}^{3}$ be absolutely isolated. If $E_{j}$ in the data $\left(^{*}\right)$ is created by blowing-up a curve then, except for the situation described by figure 5.12, we have that $\mathcal{R}=0$.

Proof. - When the curve $D$ is not projective it cannot belong to any projective exceptional surface. One can then verify that only the intersection configurations of Proposition 5.1 can occur on $E^{0}$. (In fact blowingups with a non-projective curve as center are superfluous here.) So we only have to show that the second exceptional situation of THEOREM 5.9 cannot occur in this case. This is done in Proposition 5.12 below.

Proposition 5.12. - Let all singular points of the surface $Y$ in $\mathbb{A}^{3}$ be absolutely isolated. If the exceptional surface $E_{j}$ is created as a ruled surface $E^{0}$ over a curve $D$ of genus 1 , then the (reduced) intersections $C_{i}$, $i \in T$, on $E^{0}$ cannot consist of three nonintersecting curves with associated number $d=1$.

Proof. - We use the same notations as in Proposition 4.0. The exceptional situation described in the theorem implies that the curve $D$ lies in $Y^{(1)}$ and in two previously created surfaces $E_{i}^{(1)}$, and that $D$ intersects no other exceptional surfaces, since those would cause fibers to appear in the intersection configuration on $E^{0}$. Now such a situation can only occur if, after a first blowing-up of the surface $Y$ in some singular point $P$, the intersection $E_{1} \cap Y^{*}$ contains a nonsingular curve $C$ of genus 1 . Here $E_{1}$ and $Y^{*}$ denote respectively the created exceptional surface and the strict transform of $Y$ by this blowing-up. (It is not difficult to verify that all other possibilities yield fibers on $E^{0}$.) So (if we take $P$ as the origin of $\mathbb{A}^{3}$ ) the equation $f(x, y, z)=0$ of $Y$ in $\mathbb{A}^{3}$ must be

$$
Y: g_{\ell}(x, y, z) g_{3}(x, y, z)^{n}+g_{\ell+3 n+1}(x, y, z)+\sum_{i \geq 2} g_{\ell+3 n+i}(x, y, z)=0,
$$

where the $g_{i}$ are homogeneous polynomials of degree $i$. In one of the three affine charts of the embedding space we can write the equation of $E_{1}$ 
and $Y^{*}$ as

$$
E_{1}: x=0
$$

and

$$
Y^{*}: g_{\ell}(1, y, z) g_{3}(1, y, z)^{n}+x g_{\ell+3 n+1}(1, y, z)+\sum_{i \geq 2} x^{i} g_{\ell+3 n+i}(1, y, z)=0 \text {. }
$$

Since $D$ must be part of two surfaces $E_{i}^{(1)}$ at a later stage of the resolution process, $E_{1} \cup Y^{*}$ cannot have normal crossings in the generic point of $C$; so we must have that $n \geq 2$. This implies that $g_{\ell+3 n+1}(x, y, z) \not \equiv 0$ for otherwise the generic point of $C$ would be singular on $Y^{*}$, which contradicts that $Y$ has only absolutely isolated singularities. Now to encounter $D$ as described above in some next step of the resolution process all points of $C$ must be regular points of $Y^{*}$ (otherwise we again get fibers on $\left.E^{0}\right)$, and so the polynomials $g_{3}(1, y, z)$ and $g_{\ell+3 n+1}(1, y, z)$ cannot have common roots.

Combining this with the same result for the two other affine charts we obtain that $g_{3}(x, y, z)=g_{\ell+3 n+1}(x, y, z)=0$ has no solutions in $\mathbb{P}^{2}$, which is of course impossible.

To conclude this section we prove that if $E_{j}$ in the data $\left(^{*}\right)$ is created as a ruled surface over a projective curve of genus $g \geq 2$, then the only possible intersection configurations on $E^{0}$ consist of the (simple) configurations of the Propositions 5.1 and 5.3.

Proposition 5.13. - Let $\Pi: E \rightarrow D_{0}$ be a ruled surface over a nonsingular projective curve $D_{0}$ of genus $g \geq 2$. Let $C_{i}^{\prime}, 1 \leq i \leq t$, be curves on $E$ with associated number $d_{i}>0$, and let $C_{j}, 1 \leq j \leq k$, be fibers of $\Pi(t \geq 2, k \geq 0)$. Set $\stackrel{\circ}{E}=E \backslash\left[\left(\bigcup_{1 \leq i \leq t} C_{i}^{\prime}\right) \cup\left(\bigcup_{1 \leq j \leq k} C_{j}\right)\right]$. If $\chi(\stackrel{\circ}{E}) \leq 0$ then $t=2, d_{1}=d_{2}=1$, and each point of $C_{1}^{\prime} \cap C_{2}^{\prime}$ belongs also to some fiber $C_{j}$ (Fig. 5.5), so in fact $\chi(\stackrel{\circ}{E})=0$.

Proof. - Let $g_{i}$ be the genus of $C_{i}^{\prime}$ for $i=1, \ldots, t$; then

$$
\chi\left(C_{i}^{\prime}\right)=2-2 g_{i}-x_{i}
$$

for some $x_{i} \geq 0$. Let the curve $C_{i}^{\prime}$ intersect the fiber $C_{j}$ in $d_{i j}$ points, so $1 \leq d_{i j} \leq \bar{d}_{i}$. And let finally $C_{i}^{\prime}$ intersect $\left(\bigcup_{\ell \neq i} C_{\ell}^{\prime}\right) \backslash\left(\bigcup_{1 \leq j \leq k} C_{j}\right)$ in $s_{i}$ points. Then

$$
\chi(\stackrel{\circ}{E})=2(2-2 g)-\left[\sum_{i=1}^{t}\left(2-2 g_{i}-x_{i}-\sum_{j=1}^{k} d_{i j}-s_{i}\right)+2 k+s\right],
$$

TOME $121-1993-\mathrm{N}^{\circ} 4$ 
where $2 s \leq \sum_{i=1}^{t} s_{i}$. So :

$$
\chi(\stackrel{\circ}{E})=2(2-2 g)+\sum_{i=1}^{t}\left(2 g_{i}-2+x_{i}\right)+\sum_{j=1}^{k}\left(\sum_{i=1}^{t} d_{i j}-2\right)+\sum_{i=1}^{t} s_{i}-s .
$$

- We first suppose that $t \geq 3$. Then :

$$
\begin{aligned}
\chi(\stackrel{\circ}{E})=\underbrace{2\left(g_{1}-g\right)}+\underbrace{x_{1}}+\underbrace{2\left(g_{2}-g\right)} & +\underbrace{x_{2}}+\sum_{i=3}^{t}\left(2 g_{i}-2+x_{i}\right) \\
& +\underbrace{\sum_{j=1}^{k}\left(\sum_{i=1}^{t} d_{i j}-2\right)}+\underbrace{\sum_{i=1}^{t} s_{i}-s} .
\end{aligned}
$$

Since $g_{i} \geq g$ for all $i=1, \ldots, t$ the underbraced terms are positive, and the remaining sum is strictly positive. So we never have that $\chi(\stackrel{\circ}{E}) \leq 0$.

- We now suppose that $t=2$. Then :

$$
\chi(\stackrel{\circ}{E})=\underbrace{2\left(g_{1}-g\right)}+\underbrace{x_{1}}+\underbrace{2\left(g_{2}-g\right)}+\underbrace{x_{2}}+\underbrace{\sum_{j=1}^{k}\left(d_{1 j}+d_{2 j}-2\right)}+\underbrace{s},
$$

since in this case $s_{1}=s_{2}=s$. Again all underbraced terms are positive, so they must all be zero to allow that $\chi(\stackrel{\circ}{E}) \leq 0$. We then have $g_{1}=g_{2}=g$. By considering the natural map from a desingularization of $C_{i}$ to $D_{0}$ we have Hurwitz's theorem

$$
2 g_{i}-2=d_{i}(2 g-2)+R_{i}
$$

for $i=1$ and 2 , where $R_{i} \geq 0$. It implies that $d_{1}=d_{2}=1$. But then necessarily $C_{1}^{\prime} \cong C_{2}^{\prime} \cong D_{0}$ and thus $x_{1}=x_{2}=0$ and $d_{1 j}=d_{2 j}=1$ for all $j=1, \ldots, k$.

So to make $\chi(\stackrel{\circ}{E})=0$ we only have to require that $s=0$. This is the situation described in the proposition.

Remark 5.14. - We did not study the case $t=1$ in Proposition 5.13 because a projective curve $D$ can only be the center of a blowing-up in the global resolution process of $Y$ if it lies in at least one exceptional surface, and then the intersection of $E^{0}$ with (the strict transform of) this surface has associated number $d=1$. This is an example of Proposition 5.1 (ii). 
Remark 5.15. - If we put $g=1$ in the data of Proposition 5.13 and still require that $\chi(\stackrel{\circ}{E}) \leq 0$, then the same configurations as for $g \geq 2$ are possible, together with a number of «problem» cases. We do not know if those «problem » configurations can really occur on $E^{0}$, but if they do occur we have that $\mathcal{R}$ is not identically zero (as rational function in $q$ ).

\section{Higher dimensions}

In this section we treat some examples of the data $\left(^{*}\right)$ in higher dimensions. Extending the notations of paragraph 2, we suppose in the Propositions 6.1 and 6.5 that $E^{0} \cong E_{j}$ and in the Propositions 6.2 and 6.4 that $E_{j}$ is obtained from $E^{0}$ by one blowing-up. (If we generalize Proposition 2.3 to higher dimensions these assumptions are superfluous.)

Let $x_{0}, \ldots, x_{n}$ denote homogeneous coordinates in $\mathbb{P}^{n}(n \geq 2)$.

Proposition 6.1. - Suppose that $E^{0} \cong \mathbb{P}^{n}$. Let card $T=k+1$ where $1 \leq k \leq n$, and let $C_{i}$ be the hyperplane $x_{i}=0$ for $i=0, \ldots, k$. Then

(i) $\chi(\stackrel{\circ}{E})=0$ and

(ii) $\mathcal{R}=0$.

Proof.

(i) We proceed by double induction on $n$ and $k$, and denote the corresponding $\chi(\stackrel{\circ}{E})$ by $\chi_{k}^{n}$. For $n=2$ the statement is trivial. For arbitrary $n \geq 3$ we have that $\chi_{1}^{n}=n+1-2 n+(n-1)=0$. And for $k \geq 2$ we can easily see that

$$
\chi_{k}^{n}=\chi_{k-1}^{n}-\chi_{k-1}^{n-1}
$$

which is zero by induction.

(ii) Again proceeding by double induction on $n$ and $k$ we will show that the corresponding contribution $\mathcal{R}_{k}^{n}$ can be expressed as

$$
\mathcal{R}_{k}^{n}=\frac{(q-1)^{k}\left(q^{\sum_{i=0}^{k}\left(\alpha_{i}-1\right)+n+1}-1\right)}{\prod_{i=0}^{k}\left(q^{\alpha_{i}}-1\right)},
$$

which then equals zero by relation (B1). For $n=2$ this can easily be verified. For arbitrary $n \geq 3$ we have that

$$
\begin{aligned}
& \begin{aligned}
\mathcal{R}_{1}^{n}=\left(q^{n-2}+q^{n-3}+\ldots+q+1\right) & \frac{(q-1)^{2}}{\left(q^{\alpha_{0}}-1\right)\left(q^{\alpha_{1}}-1\right)} \\
& \quad+q^{n-1}\left(\frac{q-1}{q^{\alpha_{0}}-1}+\frac{q-1}{q^{\alpha_{1}}-1}\right)+q^{n-1}(q-1)
\end{aligned} \\
& \quad=\frac{(q-1)\left(q^{\alpha_{0}+\alpha_{1}+n-1}-1\right)}{\left(q^{\alpha_{0}}-1\right)\left(q^{\alpha_{1}}-1\right)} . \\
& \text { томе } 121-1993-\mathrm{N}^{\circ} 4
\end{aligned}
$$



And for $k \geq 2$, since $\mathcal{R}_{k}^{n}=\mathcal{R}_{k-1}^{n}-\mathcal{R}_{k-1}^{n-1}+\frac{q-1}{q^{\alpha_{k}}-1} \mathcal{R}_{k-1}^{n-1}$, we have by
induction that :

$$
\begin{aligned}
\mathcal{R}_{k}^{n} & =(q-1)^{k-1}\left[\frac{q^{\sum_{i=0}^{k-1}\left(\alpha_{i}-1\right)+n+1}-1}{\prod_{i=0}^{k-1}\left(q^{\alpha_{i}}-1\right)}+\frac{\left(q-q^{\alpha_{k}}\right)\left(q^{\sum_{i=0}^{k-1}\left(\alpha_{i}-1\right)+n}-1\right)}{\left(q^{\alpha_{k}}-1\right) \prod_{i=0}^{k-1}\left(q^{\alpha_{i}}-1\right)}\right] \\
& =\frac{(q-1)^{k}\left(q^{\sum_{i=0}^{k}\left(\alpha_{i}-1\right)+n+1}-1\right)}{\prod_{i=0}^{k}\left(q^{\alpha_{i}}-1\right)} \cdot \square
\end{aligned}
$$

Proposition 6.2. - Suppose that $E^{0} \cong \mathbb{P}^{n}$ and that $n$ is even. Let card $T=k$ where $k \geq 3$, and let $C_{i}$ be the hyperplane $x_{1}+\lambda_{i} x_{0}=0$ for $i=1, \ldots, k$ where all $\lambda_{i}$ are different (Fig. 6.1). So $\chi(\stackrel{\circ}{E})=2-k$. Then $\mathcal{R}=0$.

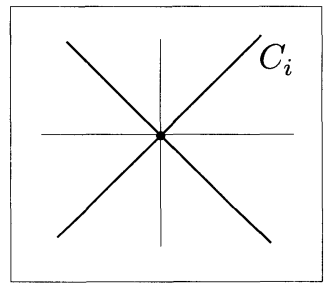

Figure 6.1

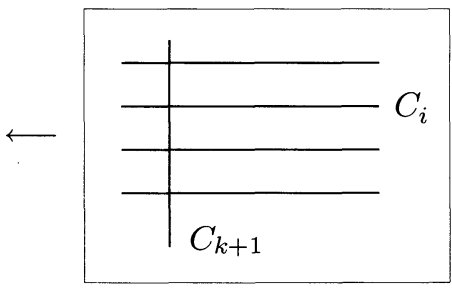

Figure 6.2

Proof. - We obtain an embedded resolution of $\bigcup_{1<i<k} C_{i}$ in $\mathbb{P}^{n}$ by constructing one blowing-up with center $x_{0}=x_{1}=0$ and exceptional variety $C_{k+1}$, which is isomorphic to a projective bundle over $\mathbb{P}^{n-2}$ with fibers isomorphic to $\mathbb{P}^{1}$ (Fig. 6.2). Then :

$$
\begin{aligned}
\mathcal{R}=\left(q^{n-2}+q^{n-3}+\ldots+q+1\right) \sum_{i=1}^{k} \frac{(q-1)^{2}}{\left(q^{\alpha_{i}}-1\right)\left(q^{\alpha_{k+1}}-1\right)} \\
+(q+1-k)\left(q^{n-2}+q^{n-3}+\ldots+q+1\right) \frac{(q-1)}{q^{\alpha_{k+1}}-1} \\
+q^{n-1} \sum_{i=1}^{k} \frac{q-1}{q^{\alpha_{i}}-1}+q^{n-1}(q+1-k) \\
=\frac{\left(q^{\alpha_{k+1}+n-1}-1\right)}{q^{\alpha_{k+1}}-1}\left[q+1-k+(q-1) \sum_{i=1}^{k} \frac{1}{q^{\alpha_{i}}-1}\right] .
\end{aligned}
$$


Now relation (B1) and relation (A) yield respectively

$$
\sum_{i=1}^{k}\left(\alpha_{i}-1\right)+n+1=0 \quad \text { and } \quad \alpha_{\mathrm{k}+1}=\sum_{\mathrm{i}=1}^{\mathrm{k}}\left(\alpha_{\mathrm{i}}-1\right)+2
$$

implying that $\alpha_{k+1}+n-1=0$ and thus also that $\mathcal{R}=0$.

REMARK 6.3. - For odd $n$ we have of course the same result; so these are examples where $\mathcal{R}=0$ when we don't « expect» it.

Proposition 6.4. - Suppose that $E^{0} \cong \mathbb{P}^{3}$. Let $\operatorname{card} T=2$, let $C_{1}$ be the hyperplane $x_{0}=0$ and $C_{2}$ the quadric hypersurface $x_{0} x_{1}-x_{2} x_{3}=0$ $\left(C_{2} \cong \mathbb{P}^{1} \times \mathbb{P}^{1}\right)$. So $\chi(\stackrel{\circ}{E})=0$. Then $\mathcal{R}=0$.

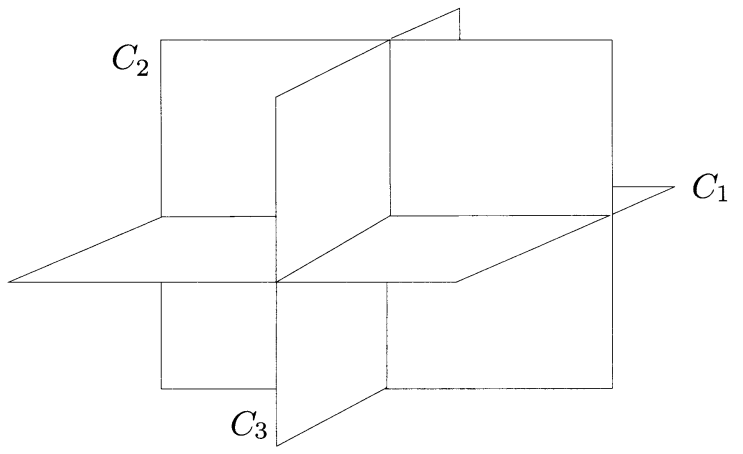

Figure 6.3

Proof. - One can check by an easy calculation that an embedded resolution of $C_{1} \cup C_{2}$ in $\mathbb{P}^{3}$ is obtained by blowing-up $\mathbb{P}^{3}$ with center $x_{0}=x_{2}=0$. Let $C_{3}$ denote the exceptional variety, which is a ruled surface over $\mathbb{P}^{1}$ (Fig. 6.3). Then :

$$
\begin{aligned}
& \begin{array}{l}
\mathcal{R}=1 \frac{(q-1)^{3}}{\prod_{i=1}^{3}\left(q^{\alpha_{i}}-1\right)}+q \sum_{\substack{i, j=1 \\
i<j}}^{3} \frac{(q-1)^{2}}{\left(q^{\alpha_{i}}-1\right)\left(q^{\alpha_{j}}-1\right)} \\
\quad+q(q-1) \frac{q-1}{q^{\alpha_{1}}-1}+q^{2} \frac{q-1}{q^{\alpha_{2}}-1}+q^{2} \frac{q-1}{q^{\alpha_{3}}-1}+q^{2}(q-1) \\
=(q-1) \frac{q^{\alpha_{2}+\alpha_{3}+1}-1}{\left(q^{\alpha_{2}}-1\right)\left(q^{\alpha_{3}}-1\right)}\left[q+\frac{q-1}{q^{\alpha_{1}}-1}\right] .
\end{array} \\
& \text { тОМе } 121-1993-\mathrm{N}^{\circ} 4
\end{aligned}
$$


The relations (B1) and (A) yield respectively

$$
\left(\alpha_{1}-1\right)+2\left(\alpha_{2}-1\right)+4=0 \quad \text { and } \quad \alpha_{3}=\left(\alpha_{1}-1\right)+\left(\alpha_{2}-1\right)+2 \text {, }
$$

implying that $\alpha_{2}+\alpha_{3}+1=0$, and thus that $\mathcal{R}=0$.

For an arbitrary projective space bundle we can also associate a number $d \in \mathbb{N}$ to any prime divisor (see Theorem 0.1 ).

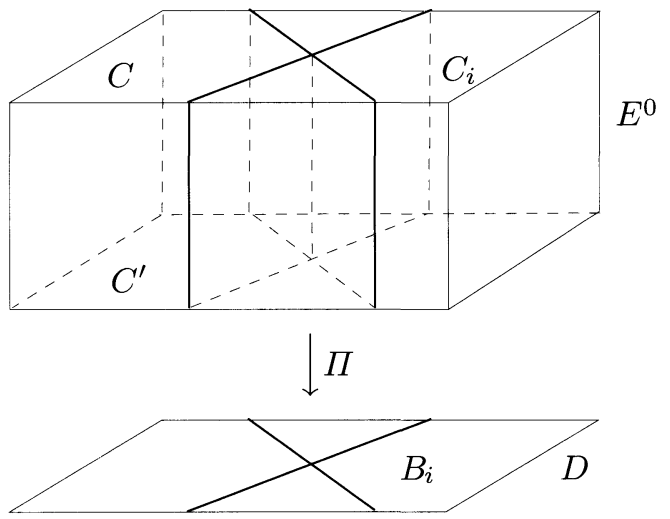

Figure 6.4

Proposition 6.5. - Let $E^{0}$ be a projective space bundle $\Pi: E^{0} \rightarrow D$ over a nonsingular variety $D$ with fibers isomorphic to $\mathbb{P}^{1}$ and let card $T=k+2$ where $k \geq 0$. Let $C$ and $C^{\prime}$ have associated numbers $d=d^{\prime}=1$ and $C \cap C^{\prime}=\emptyset$, and let $C_{i}, 1 \leq 1 \leq k$, be the inverse images $\Pi^{-1} B_{i}$ of nonsingular varieties $B_{i}$ in $D$ such that $\bigcup_{1 \leq i \leq k} B_{i}$ has only normal crossings on $D$ (Fig. 6.4). So $\chi(\stackrel{\circ}{E})=0$. Then $\mathcal{R}=0$.

Proof. - Let $\bar{D}(\bar{K})$ denote the set of rational points over $\bar{K}$ of the reduction modulo $\mathfrak{p} R_{\mathfrak{p}}$ of $D$ [D1, $\left.\S 2\right]$. Then we can write $\mathcal{R}$ as :

$$
\sum_{P \in \bar{D}(\bar{K})}\left(q-1+\frac{q-1}{q^{\alpha}-1}+\frac{q-1}{q^{\alpha^{\prime}}-1}\right) \prod_{\substack{1 \leq i \leq k \\ P \in \bar{B}_{i}}} \frac{q-1}{q^{\alpha_{i}}-1} .
$$

Since $\alpha+\alpha^{\prime}=0$ by relation (B1) we obtain that $\mathcal{R}=0$. 


\section{Appendix}

Proof of proposition 4.0. - Let $X^{(1)}$ and $X^{(2)}$ respectively denote the embedding space of the resolution $h$ before and after the blowing-up with center $D$, and set $r=\operatorname{codim}\left(D, X^{(1)}\right)$.

(i) Let $P$ be any point of $D$ and let $\widehat{\mathcal{O}}=\widehat{\mathcal{O}}_{X^{(1)}, P}$ denote the completed local ring of $P$ on $X^{(1)}$. We can choose a regular system of parameters $t_{1}, \ldots, t_{n+1}$ in $\widehat{\mathcal{O}}$ such that the ideal in $\widehat{\mathcal{O}}$ of $D$ is generated by $t_{1}, \ldots, t_{r}$. Since $\widehat{\mathcal{O}}$ is a formal power series ring in $t_{1}, \ldots, t_{n+1}$ and the multiplicity of the generic point of $D$ on $Y^{(1)}$ is $\mu$, the ideal of $Y^{(1)}$ in $\widehat{\mathcal{O}}$ is generated by an element

$$
f_{\mu}\left(x_{1}, \ldots, x_{r}\right)+\sum_{i>0} f_{\mu+i}\left(x_{1}, \ldots, x_{r}\right)
$$

where the $f_{i}$ are homogeneous polynomials of degree $i$ in $x_{1}, \ldots, x_{r}$ with coefficients in $k\left[\left[x_{r+1}, \ldots, x_{n+1}\right]\right]$. Moreover, since the multiplicity of $P$ on $Y^{(1)}$ is $\mu$, at least one coefficient of $f_{\mu}$ does not belong to the maximal ideal $\left(x_{r+1}, \ldots, x_{n+1}\right)$ of $k\left[\left[x_{r+1}, \ldots, x_{n+1}\right]\right]$.

By definition of blowing-up we can describe in the chart $\left\langle x_{1} \neq 0 »\right.$ the completed local ring $\widehat{\mathcal{O}}_{Q}=\widehat{\mathcal{O}}_{X^{(2)}, Q}$ of any point $Q$ of $F=\Pi^{-1}\{P\}$ as $k\left[\left[x_{1}, y_{2}, \ldots, y_{r}, x_{r+1}, \ldots, x_{n+1}\right]\right]$, where the ideal in $\widehat{\mathcal{O}}_{Q}$ of $E^{0}$ is generated by $x_{1}$ and the ideal of $Y^{(2)}$ is generated by

$$
f_{\mu}\left(1, y_{2}+c_{2}, \ldots, y_{r}+c_{r}\right)+\sum_{i>0} x_{1}^{i} f_{\mu+i}\left(1, y_{2}+c_{2}, \ldots, y_{r}+c_{r}\right)
$$

for some $\left(c_{2}, \ldots, c_{r}\right) \in k^{r-1}$. Since the ideal in $\widehat{\mathcal{O}}_{Q}$ of the fiber $F$ is generated by $x_{1}, x_{r+1}, \ldots, x_{n+1}$ we can describe the completed local ring $\widehat{\mathcal{O}}_{F, Q}$ of $Q$ on $F \cong \mathbb{P}^{r-1}$ as $k\left[\left[y_{2}, \ldots, y_{r}\right]\right]$. Then the ideal in $\widehat{\mathcal{O}}_{F, Q}$ of $\left(Y^{(2)} \cap E^{0}\right) \cap F$ is generated by

$$
\tilde{f}_{\mu}\left(1, y_{2}+c_{2}, \ldots, y_{r}+c_{r}\right)
$$

where $\tilde{f}_{\mu}$ is obtained from $f_{\mu}$ by substituting $x_{r+1}=\ldots=x_{n+1}=0$ and is not identically zero.

Combining this with the analogous results for the other charts we obtain that the intersection of $Y^{(2)} \cap E^{0}$ with the fiber $F \cong \mathbb{P}^{r-1}$ is described (in homogeneous coordinates $y_{1}, \ldots, y_{r}$ ) by $\tilde{f}_{\mu}\left(y_{1}, \ldots, y_{r}\right)=0$, implying that $\sum_{i=1}^{s} d_{i} \leq \mu$. Moreover we see that no fiber of $\Pi$ is part of $Y^{(2)} \cap E^{0}$ and consequently $d_{i} \geq 1$ for all $i=1, \ldots, s$.

(ii) Replacing $Y^{(1)}$ by $E_{\ell}^{(1)}$ in (i) the conclusion is evident.

TOME $121-1993-\mathrm{N}^{\circ} 4$ 
Remark. - If $D$ is a point in Proposition 4.0 we have in the proof that $r=n+1, \tilde{f}_{\mu}=f_{\mu}$ and $F=E \cong \mathbb{P}^{n}$. Then we can describe the irreducible components $C_{i}$ of $Y^{(2)} \cap E^{0}$ as

$$
G_{i}\left(y_{1}, \ldots, y_{n+1}\right)=0
$$

where $f_{\mu}=\prod_{i=1}^{s} G_{i}^{m_{i}}$ is the decomposition in irreducible components of $f_{\mu}$ and $G_{i}$ has degree $d_{i}$ for $i=1, \ldots, s$.

\section{BIBLIOGRAPHY}

[A] A'CAmpo (N.). - La fonction zeta d'une monodromie, Comment. Math. Helv., t. 50, 1975, p. 233-248.

[D1] Denef (J.). - On the degree of Igusa's local zeta function, Amer. J. Math., t. 109, 1987, p. 991-1008.

[D2] Denef (J.). - Local Zeta Functions and Euler Characteristics, Duke Math. J., t. 63,3, 1991, p. 713-721.

[EH] Eisenbud (D.) and Harris (J.). - Divisors on general curves and cuspidal rational curves, Invent. Math., t. 74, 1983, p. 371-418.

[Ha] Hartshorne (R.). - Algebraic Geometry. — Springer Verlag, 1977.

[Hi] Hironaka (H.). - Resolution of singularities of an algebraic variety over a field of characteristic zero, Ann. Math., t. 79, 1964, p. 109-326.

[Ig1] IGusA (J.). - Complex powers and asymptotic expansions (I), J. Reine Angew. Math., t. 268/269, 1974, p. 110-130. (II) ibid., t. 278/279 1975, p. 307-321.

[Ig2] Igusa (J.). - Lectures on Forms of Higher degree. - Narosa Publ. House, New Delhi, 1978.

[Ig3] IgusA (J.). - Complex powers of irreducible algebroid curves, in Geometry today, Roma 1984, Progress in Mathematics 60, Birkhaüser 1985 , p. 207-230.

[L1] Loeser (F.). - Fonctions d'Igusa p-adiques et polynômes de Bernstein, Amer. J. Math., t. 110, 1988, p. 1-21.

[L2] Loeser (F.). - Fonctions zêta locales d'Igusa à plusieurs variables, intégration dans les fibres et discriminants, Ann. Sci. École Norm. Sup., t. 4, 22, 1989, p. 435-471. 
[L3] Loeser (F.). - Fonctions d'Igusa p-adiques, polynômes de Bernstein et polyèdres de Newton, J. Reine Angew. Math., t. 412, 1990, p. 75-96.

[Ma1] Malgrange (B.). - Intégrales asymptotiques et monodromie, Ann. Sci. École Norm. Sup., t. 4, 7, 1974, p. 405-430.

[Ma2] Malgrange (B.). - Polynômes de Bernstein-Sato et cohomologie évanescente, Astérisque, t. 101/102, 1983, p. 243-267.

[Me1] Meuser (D.). - On the poles of a local zeta function for curves, Invent. Math., t. 73, 1983, p. 445-465.

[Me2] Meuser (D.). - The meromorphic continuation of a zeta function of Weil and Igusa type, Invent. Math., t. 85, 1986, p. 493-514.

[Mi] Milnor (J.).- Singular points of complex hypersurfaces. - Princeton Univ. Press, 1968.

[S] Strauss (L.). - Poles of a two variable $p$-adic complex power, Trans. Amer. Math. Soc., t. 278, 2, 1983, p. 481-493.

[Si] Siersma (D.). - Variation mappings on singularities with a 1dimensional critical locus, Topology, t. 30, 3, 1991, p. 445-469.

[V1] Veys (W.). - On the poles of Igusa's local zeta function for curves, J. London Math. Soc., t. 41, 2, 1990, p. 27-32.

[V2] Veys (W.). - Relations between numerical data of an embedded resolution, Amer. J. Math., t. 113, 1991, p. 573-592.

[V3] Veys (W.). - Congruences for numerical data of an embedded resolution, Compositio Math., t. 80, 1991, p. 151-169. 